\title{
Designing Isothermal Batch Deracemization Processes With Optimal Productivity: I. Parametric Analysis Using A Population Balance Equation Model
}

DOI:

10.1021/acs.cgd.9b01581

\section{Document Version \\ Accepted author manuscript}

Link to publication record in Manchester Research Explorer

Citation for published version (APA):

Vetter, T. (2020). Designing Isothermal Batch Deracemization Processes With Optimal Productivity: I. Parametric Analysis Using A Population Balance Equation Model. Crystal Growth \& Design.

https://doi.org/10.1021/acs.cgd.9b01581

Published in:

Crystal Growth \& Design

\section{Citing this paper}

Please note that where the full-text provided on Manchester Research Explorer is the Author Accepted Manuscript or Proof version this may differ from the final Published version. If citing, it is advised that you check and use the publisher's definitive version.

\section{General rights}

Copyright and moral rights for the publications made accessible in the Research Explorer are retained by the authors and/or other copyright owners and it is a condition of accessing publications that users recognise and abide by the legal requirements associated with these rights.

\section{Takedown policy}

If you believe that this document breaches copyright please refer to the University of Manchester's Takedown Procedures [http://man.ac.uk/04Y6Bo] or contact uml.scholarlycommunications@manchester.ac.uk providing relevant details, so we can investigate your claim.

\section{OPEN ACCESS}




\title{
Designing Isothermal Batch Deracemization
} Processes With Optimal Productivity: I.

\section{Parametric Analysis Using A Population Balance Equation Model}

\author{
Thomas Vetter* \\ University of Manchester, Department of Chemical Engineering and Analytical Science, M13 \\ 9PL Manchester, United Kingdom \\ E-mail: thomas.vetter@manchester.ac.uk \\ Phone: +44161306 4370
}

\begin{abstract}
Isothermal batch deracemization (Viedma ripening) is an attractive process variant to separate conglomerate forming enantiomers. These processes rely on a complex interplay between a racemization reaction in the liquid phase and crystal growth/dissolution, agglomeration and breakage in the solid phase. While reports in the literature have shown the applicability of Viedma ripening to a variety of substances, a comprehensive investigation on how to obtain maximum productivity from such processes is so far missing. This contribution introduces a novel operating protocol based on a series of batches, wherein part of the product of one batch is used to generate an initial solid phase enantiomeric excess in the next batch. It is shown that initial enantiomeric excess leading to maximum productivity depends on the kinetics involved in Viedma ripening, as well as process parameters, such as the suspension density. This relationship is
\end{abstract}


explored using a parametric sensitivity analysis carried out on a process model based on dimensionless population balance equations. The general trends identified from the parametric analysis highlight that processes with maximum productivity should be carried out at i) high breakage intensities, ii) low agglomeration intensities, and iii) high suspension densities. The initial enantiomeric excess necessary to reach maximum productivity varies strongly with the kinetics of the different phenomena involved and falls within a wide range of $25-80 \%$.

\section{Introduction}

Manufacturing pure enantiomers is critically important in a variety of industries and can in general be accomplished by starting from a chirally pure starting material (from the chiral pool existing in nature), through asymmetric synthesis or by separation of enantiomers. For the latter there are a variety of processes available ${ }^{1}$; among them crystallization. Classically, the separation of enantiomers using crystallization is carried out by diastereomeric resolution, i.e., the formation of a salt or co-crystal with a chiral counter ion/co-former. If the salts/cocrystals of the two enantiomers exhibit sufficiently different solubility, they can be separated in a conventional crystallization process. However, this requires the availability of such a chiral resolving agent, as well as the existence of an appropriate salt or co-crystal. For conglomerate forming systems of enantiomers, i.e., systems where each enantiomer occurs individually in separate crystals but never mixed in the same, preferential crystallization has been shown to be an attractive alternative ${ }^{2}$. A variety of isothermal and polythermal operating modes carried out in batch crystallizers have been reported ${ }^{3,4}$, as well as processes running in continuous mode ${ }^{5,6}$. If only one enantiomer is the desired product, such processes deliver at best a yield of $50 \%$, i.e., all molecules of the undesired enantiomer are waste, while the molecules of the desired enantiomer can be harvested. If racemization reactions are available, it becomes theoretically feasible to increase the yield of the process to near $100 \%$ through a combination of resolution, racemization and recycling steps ${ }^{1,7}$. 
In a groundbreaking work, $\mathrm{Viedma}^{8}$ reported a process that delivered enantiopure sodium chlorate crystals starting from an initially slightly enriched mixture. ${ }^{\text {a }}$ The process involved grinding the crystals isothermally in the presence of glass beads (providing a means of attrition) and at solution conditions near the thermodynamic equilibrium (cf. Figure 1). It was subsequently shown that this process can be applied to chiral organic substances that can be racemized in solution, leading to enantiomerically pure solid products ${ }^{9}$. The process therefore enables complete enantiomeric resolution from an only slightly enriched solid phase in a single processing step, rather than the multi-step resolution, racemization, recycle process mentioned above.

The prerequisites for such Viedma ripening processes are that the pair of enantiomers is conglomerate forming and that they can be racemized in solution (using a suitable catalyst if necessary). While these restrictions are limiting the number of substances that can undergo Viedma ripening, crystal engineering approaches have been employed to extend is applicability to substances forming racemic compounds (e.g., through the formation of conglomerate forming co-crystals, salts ${ }^{10}$, etc. $)^{11}$. It has been shown that Viedma ripening can be successfully conducted with achiral reactands forming chiral products through reversible reactions ${ }^{12,13}$. The process has also been shown to work at different scales ${ }^{14}$, as well as when crystal attrition was generated using different techniques ${ }^{15,16}$. b

When tracking the evolution of such processes it is instructive to consider the enantiomeric excess in the solid and the liquid phase, $E$ and $e$, which can be defined as:

$$
E=\frac{m_{\mathrm{D}}-m_{\mathrm{L}}}{m_{\mathrm{D}}+m_{\mathrm{L}}} \quad e=\frac{c_{\mathrm{D}}-c_{\mathrm{L}}}{c_{\mathrm{D}}+c_{\mathrm{L}}}
$$

where $m_{i}$ is the mass of crystals of enantiomer $i$ in the crystallizer and $c_{i}$ is the enantiomer's concentration in the liquid phase. The solid phase enantiomeric excess against time curve

\footnotetext{
${ }^{a} \mathrm{NaClO}_{3}$ is achiral in solution, but crystallizes in chiral crystal structures; the enrichment therefore was in the solid phase

${ }^{b}$ Polythermal methods, i.e., processes employing temperature cycles or multiple communicating vessels held at different temperatures to accomplish deracemization, have also been developed ${ }^{17-20}$, but are outside the scope of this work.
} 


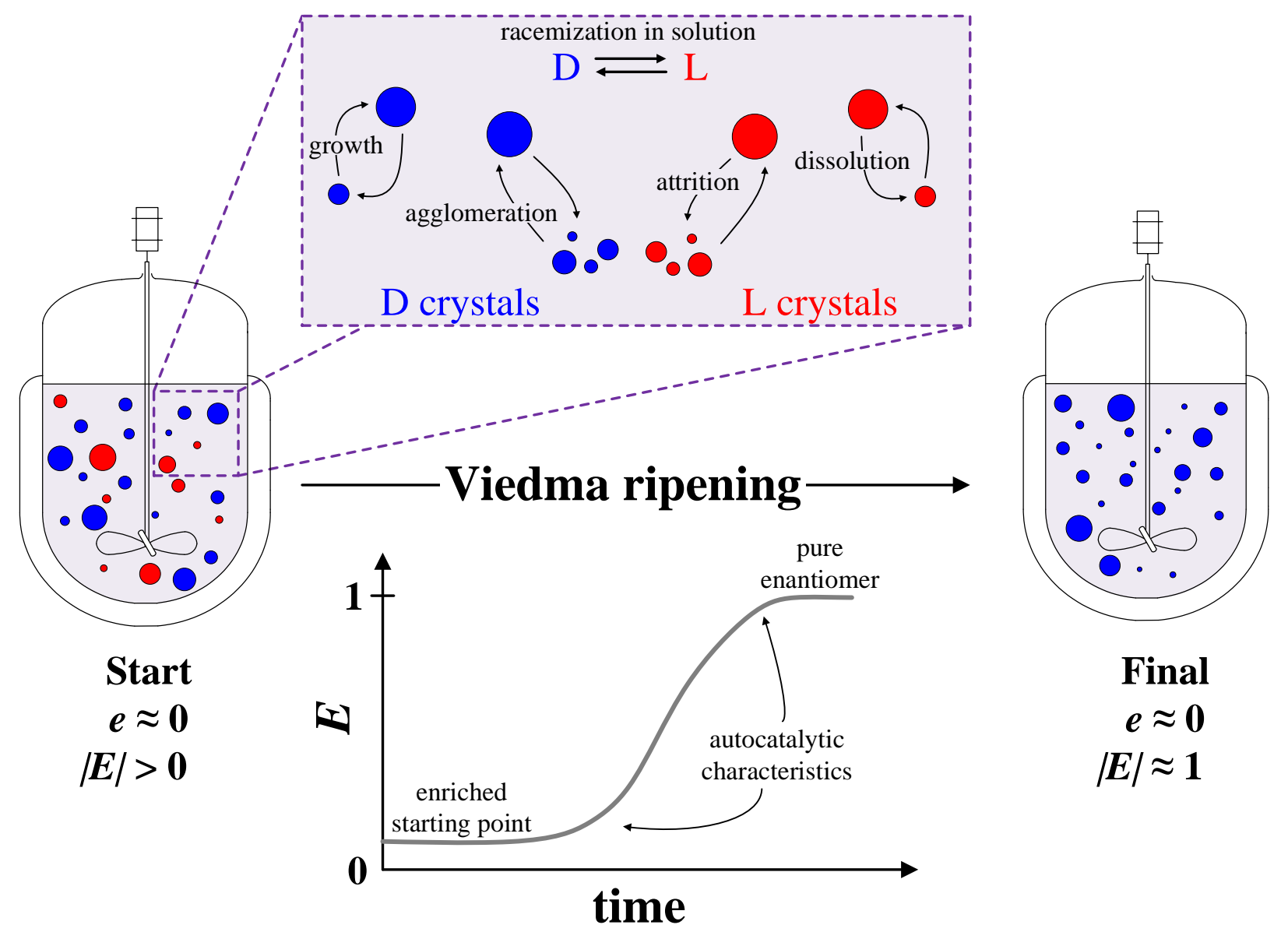

Figure 1: Illustration of an isothermal batch Viedma ripening process leading to an enantiopure solid product from a starting point that is enantiomerically enriched in the solid phase. The process proceeds through a combination of different mechanisms ${ }^{9,11,21,22}$, involving a racemization reaction in the liquid phase (converting molecules of the $\mathrm{L}$ enantiomer into molecules of the D enantiomer and vice versa and keeping the liquid phase enantiomeric excess, $e$, typically close to zero), and a combination of crystal growth, dissolution (Ostwald ripening), agglomeration, and attrition on the solid side. As a result of this complex interplay of mechanisms the process typically exhibits autocatalytic characteristics when the evolution of the enantiomeric excess in the solid phase $(E)$ is tracked over time, i.e., it proceeds initially slow, then faster and then slow again upon nearing solid phase enantiopurity (see coordinate system). 
typically exhibits autocatalytic characteristics in a Viedma ripening process, i.e., the solid phase enantiomeric excess increases slowly at first, the increase then accelerates and finally slows down again when enantiopurity is approached (as conceptually depicted in Figure 1). Through a combination of experimental efforts and dedicated modelling work the research community has rationalized this behavior as the result of a complex interplay between Ostwald ripening (crystal growth and dissolution due to a size-dependent solubility), agglomeration and breakage affecting the crystal populations of both enantiomers, as well as the racemization reaction occurring in the liquid phase. ${ }^{9,11,16,21-23}$ A schematic overview of the Viedma ripening process and the mechanisms involved is given in Figure 1.

While this insight has been successfully used to generate a number of working examples for different compounds and process configurations as mentioned above, optimizing these processes to deliver the highest possible productivity has so far not received extensive attention. Specifically, there is no comprehensive study available that i) devises processing schemes that can be efficiently used in batch campaigns (rather than a single batch) and that ii) investigates how the optimal operating conditions employed in such a scheme change in dependence of the kinetics of the different mechanisms contributing to Viedma ripening. The goal of this work is to address this gap. It is structured as follows: in Section 2 a processing strategy to conduct whole batch campaigns in an efficient way is presented. Key processing parameters occurring in this strategy will be identified. In Section 3 a process model based on population balance equations is detailed. The process model will be made dimensionless in order to identify the smallest number of parameter groups that determine the outcome of the process and an efficient numerical technique to arrive at solutions of the model is developed. In Section 4, the process is optimized to yield maximum productivity for a wide range of possible kinetics. The operating parameters leading to the optimized productivity for each set of kinetics will be identified and trends highlighted. Finally, conclusions will be presented. 


\section{Process schemes}

Once the processing equipment and solvents are determined the single batch deracemization process depicted in Figure 1 still has a number of operating parameters that can be chosen and that influence the process outcome and productivity. These are i) the suspension density (mass of crystals per volume of suspension), ii) the size distribution of the crystal populations of both enantiomers, and iii) the initial enantiomeric excess. The interplay between size distributions of both enantiomers and initial enantiomeric excess has been explored in an extensive modelling study by Iggland et al. ${ }^{24}$. The authors have shown that both the size distribution, as well as the initial enantiomeric excess need to be controlled to achieve deracemization towards the desired enantiomer. Indeed, it was shown that when these parameters are not chosen correctly, there are instances where an initial enantiomeric excess in a desired enantiomer, say D, combined with inappropriate size distributions (e.g., average size of the target D crystals smaller than the undesired L crystals) gets overturned and the process outcome is a chirally pure product consisting of undesired L crystals.

That said, in practice the overall size distributions of the two enantiomers at the start of the process result from the combination of racemic material to be deracemized ${ }^{\mathrm{c}}$, as well as enantiomerically enriched material from the previous batch. The amount of enriched material added in this way and of newly racemic material to be converted therefore determines the initial solid phase enantiomeric excess. When considering a whole campaign of batches, i.e., multiple batch Viedma ripening processes in series, the most convenient way to provide an initial enantiomeric excess is to use part of the enantiomerically enriched product from the previous batch and mix it with new racemic material that is to be converted. Such a batch campaign where part of the product is recycled is shown in Figure 2. Similarly, the mother liquor of one batch can be used to suspend the crystals of the next batch. This has several advantages: i) if the racemization reaction occurring in the liquid phase requires a

\footnotetext{
${ }^{\mathrm{c}}$ If the processing steps carried out before the deracemization step are not chirally selective, it is reasonable to assume that the crystal size distributions of both enantiomers are identical within the racemic material added to the deracemization step.
} 
(homogeneous) catalyst, the catalyst is recycled with the mother liquor and can be re-used, ii) since new crystals are essentially suspended in an already saturated solution, there is no further product that gets dissolved into the liquid and that would ultimately be lost. In the presence of impurities in the starting material or in the case of product or catalyst degradation, a purge stream becomes necessary to avoid the build up of impurities during the batch campaign. When a purge stream is necessary or when the filtration process does not separate mother liquor from solids perfectly, a solvent make up stream (including catalyst) must be introduced as well (both purge and solvent make-up streams are drawn in gray in Figure 2). For the sake of simplicity, purge and solvent make up streams are not included in the following; however, they would represent a straightforward extension of the concepts introduced here.

One can hypothesize that such a process scheme possibly converges to a "cyclic steady state" (a terminology that is borrowed from the field of adsorption/chromatography ${ }^{25,26}$ ) after a few batches. This means that each subsequent batch will proceed through the same trajectory in time provided that the processing conditions are kept the same from batch to batch and the newly added racemic crystals have the same particle size distribution. The productivity of this operating scheme depends on the underlying kinetics of the deracemization process ${ }^{22,27}$, the solid phase enantiomeric excess at the start of the batch ${ }^{9,19,22,28}$ and the overall suspension density of the process (which is limited by practical considerations, e.g., the suspension must remain stirrable). To understand the impact of the amount of product recycled into the next batch in terms of process productivity, the following considerations are helpful: Not recycling any product would lead to no initial enantiomeric excess in the solid phase and therefore no productivity would be obtained (at least not reliably ${ }^{24}$ ). When all the product is recycled, then no new racemic crystals could be added or the targeted suspension density would be exceeded. This again would result in zero productivity. One can therefore conjecture that maximum productivity must be attained at some intermediate initial enantiomeric excess. 
The position of this maximum (or these maxima) and how it depends on the kinetics of the mechanisms contributing to Viedma ripening and substance specific parameters will be elucidated with the use of the process model detailed in the next section.

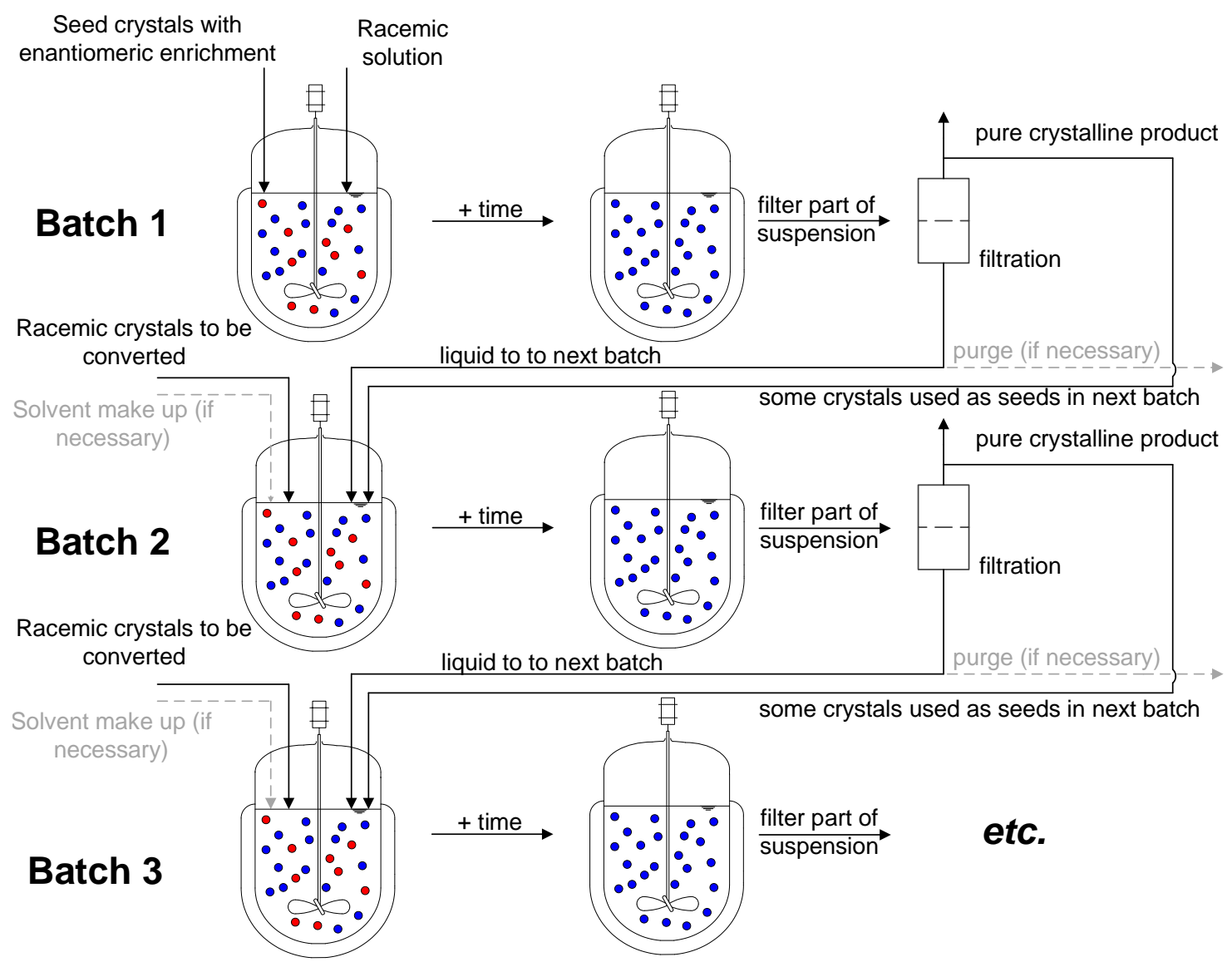

Figure 2: A conceptual optimal scheme to conduct multiple batches of an attrition-enhanced deracemization process. All of the mother liquor and part of the crystalline product of one batch is used in the next batch, leading to an efficient operation.

\section{Process model}

\subsection{Population balance equations and mass balances}

In the following a process model tracking the evolution of the solid phases and the liquid phase in the process will be described. A model for Viedma ripening must include all relevant mechanisms occurring during the process (cf. Figure 1) and must take note of the fact that 
productive crystallization processes involve a large number of crystals in the processing vessel with distributed sizes. This leads to the use of population balance equations ${ }^{29,30}$ to describe the solid phases and mass balances to describe the evolution of the liquid phase ${ }^{\mathrm{d}}$. Following Iggland and Mazzotti ${ }^{22}$ for the isothermal case and Bodàk et al. ${ }^{28}$ for the polythermal case, the relevant population balance equations can be written as

$$
\begin{aligned}
\frac{\partial n_{i}(x, t)}{\partial t}= & -\frac{\partial\left[G\left(x, c_{i}\right) n_{i}(x, t)\right]}{\partial x} \\
& +\frac{x^{2}}{2} \int_{0}^{x} \frac{A\left(\lambda, \sqrt[3]{x^{3}-\lambda^{3}}\right)}{\left(x^{3}-\lambda^{3}\right)^{2 / 3}} n_{i}(\lambda, t) n_{i}\left(\sqrt[3]{x^{3}-\lambda^{3}}, t\right) \mathrm{d} \lambda-n_{i}(x, t) \int_{0}^{\infty} A(\lambda, x) n_{i}(\lambda, t) \mathrm{d} \lambda \\
& +\int_{x}^{\infty} B(\lambda) F(\lambda, x) n_{i}(\lambda, t) \mathrm{d} \lambda-B(x) n_{i}(x, t) \quad \text { with } \quad i \in\{\mathrm{D}, \mathrm{L}\}
\end{aligned}
$$

where $n_{i}$ is the particle size distribution of enantiomer $i, t$ is time, $x$ and $\lambda$ are the particle size (length) and $A, B, F$ and $G$ are functions describing the rate of agglomeration, rate of breakage, the fragment size distribution occurring in breakage and the growth/dissolution rate of the crystals, respectively. The first line in Eq. (2) therefore represents the change of the particle size distribution over time and the growth/dissolution term, the second line represents the agglomeration terms, and the third line represents the breakage terms. The two population balance equations are coupled to material balances for the liquid phase that describe the evolution of the enantiomer concentrations over time:

$$
\frac{\mathrm{d} c_{i}}{\mathrm{~d} t}=-3 k_{\mathrm{v}} \rho_{\mathrm{c}} \int_{0}^{\infty} x^{2} G\left(x, c_{i}\right) n_{i}(x, t) \mathrm{d} x+k_{\mathrm{r}}\left(c_{j}-c_{i}\right) \quad \text { with } \quad i, j \in\{\mathrm{D}, \mathrm{L}\} \text { and } j \neq i
$$

where $c_{i}$ is the concentration of enantiomer $i, k_{\mathrm{v}}$ is a crystal shape factor relating the volume of a particle to the cube of its characteristic size, $\rho_{\mathrm{c}}$ is the density of the crystals and $k_{\mathrm{r}}$ is the racemization rate constant. The constitutive equations $(A, B$, etc.) necessary to complete

${ }^{\mathrm{d}}$ A good overview of alternative model approaches, such as Monte Carlo simulations, is given by Ricci et al. ${ }^{31}$. Simpler models with two size classes rather than a continuum of sizes, have seen some use in describing Viedma ripening ${ }^{21}$, but the choice of sizes to be modeled is an arbitrary one in such models and does not connect well to experimental evidence where particle sizes can be widely distributed. 
the model are summarized in Table 1 (note that all symbols not specifically explained in the text - mainly kinetic constants - are covered in a notation table in the supporting information). As is evident from Table 1, the model includes every mechanism involved in Viedma ripening according to the current state of the art understanding. These are: Ostwald ripening, which is modeled with a size-dependent solubility that leads to growth of large particles and dissolution of small particles at a given supersaturation, attrition/breakage, agglomeration and racemization in the liquid phase. The functional form of the constitutive equations describing the different mechanism has been discussed and justified at length elsewhere $^{22,27,28,32}$.

Table 1: Constitutive equations for the dimensional model ${ }^{\mathrm{a}, \mathrm{b}}$

\begin{tabular}{ll}
\hline mechanism & equation \\
\hline solubility & $c_{\star}(x)=c_{\infty} \exp \left(\frac{\alpha}{x}\right)$ \\
growth and dissolution & $G\left(x, c_{i}\right)=\frac{k_{\mathrm{g}}}{c_{\infty}}\left(c_{i}-c_{\star}(x)\right)$ \\
breakage & $B(x)=k_{\mathrm{b}, 1}\left(\frac{x}{x_{0}}\right)^{k_{\mathrm{b}, 2}}$ \\
& $F(\lambda, x)=3 x^{2}\left(2 k_{\mathrm{f}}+1\right)\left(\frac{2}{\lambda^{3}}\right)^{2 k_{\mathrm{f}}+1}\left(x^{3}-\frac{\lambda^{3}}{2}\right)^{2 k_{\mathrm{f}}}$ \\
& $A(\lambda, x)=\left(\frac{\lambda+x}{2}\right)^{3} \frac{k_{\mathrm{a}, 1}}{1+k_{\mathrm{a}, 2} x_{\mathrm{eq}}(\lambda, x)}$ \\
& $x_{\mathrm{eq}}(\lambda, x)=\frac{(\lambda x)^{2}}{\lambda^{2}+x^{2}-\lambda x}$ \\
\hline
\end{tabular}

${ }^{\mathrm{a}} x_{0}$ is a normalization constant, $\alpha$ is the capillary length, $c_{\star}(x)$ is the size-dependent solubility, $c_{\infty}$ is the bulk solubility (at large particle sizes), and all $k$ values are kinetic parameters. For details see the notation table in the supporting information.

${ }^{\mathrm{b}}$ In the fragment size distribution, $F(\lambda, x), \lambda$ is the size of the original particle, while $x$ represents the size of the fragment. $k_{\mathrm{f}}$ is a parameter describing the shape of the fragment size distribution, see supporting information. 


\subsection{Non-dimensionalization}

The model above contains eleven parameters in total: seven kinetic parameters $\left(k_{\mathrm{g}}, k_{\mathrm{b}, 1}, k_{\mathrm{b}, 2}\right.$, $k_{\mathrm{f}}, k_{\mathrm{a}, 1}, k_{\mathrm{a}, 2}$, and $\left.k_{\mathrm{r}}\right)$ and four substance system specific constants $\left(\alpha, c_{\infty}, k_{\mathrm{v}}\right.$, and $\left.\rho_{\mathrm{c}}\right)$. This leads to a high-dimensional parameter space that would be hard to fully explore. However, not all model parameters exhibit an independent effect on the dynamic behavior of the model. By re-casting the model into a dimensionless and normalized form, a smaller number of independent parameter groups can be identified ${ }^{22}$. This facilitates the envisioned parametric analysis of the model. To this end, normalized versions of the variables appearing in the model $\left(t, x\right.$ and $\left.c_{i}\right)$ are introduced as follows:

$$
\xi=\frac{t}{t_{0}}=\frac{3 k_{\mathrm{g}} t}{\alpha} ; \quad w=\frac{k_{\mathrm{v}} x^{3}}{v_{0}}=\frac{k_{\mathrm{v}} x^{3}}{k_{\mathrm{v}} \alpha^{3}}=\frac{x^{3}}{\alpha^{3}} ; \quad S_{i}=\frac{c_{i}}{c_{0}}=\frac{c_{i}}{c_{\infty}}
$$

where $\xi, w$, and $S_{i}$ represent a dimensionless time, a dimensionless particle volume ${ }^{\mathrm{e}}$ and a dimensionless concentration, respectively. It will be shown below that this choice of dimensionless variables has two benefits: i) it leads to the smallest possible number of independent parameter groups describing the dynamic behavior of the model and ii) it results in a growth/dissolution term that is completely parameter free (which is of interest when solving the equations numerically).

A rescaled and dimensionless particle size distribution, $f_{i}(w, \xi)$, is also introduced, which obeys the following equation ${ }^{33}$ :

$$
z f_{i}(w, \xi) \mathrm{d} w=n_{i}(x, t) \mathrm{d} x
$$

where the normalization constant $z$ with units of $\left[\mathrm{m}^{-3}\right]$ is chosen to simplify the mass balance $\mathrm{f}^{\mathrm{f}}$.

\footnotetext{
${ }^{\mathrm{e}} \mathrm{A}$ dimensionless volume is chosen instead of the dimensionless length mainly for cosmetic reasons; it allows simplifying the agglomeration term. The non-dimensionalization could similarly be done with a dimensionless length.

${ }^{\mathrm{f}}$ Note that the normalization constant $z$ was not included in the model by Iggland and Mazzotti ${ }^{22}$, which resulted in their model having a redundant parameter group, i.e., the model presented here has the same flexibility as theirs, but with one parameter group less.
} 
Defining $z$ as

$$
z=\frac{c_{\infty}}{k_{\mathrm{v}} \rho_{\mathrm{c}} \alpha^{3}}
$$

and using the definition of $w$ (provided in Eq. (4)), one therefore obtains for the dimensionless particle size distribution:

$$
f_{i}(w, \xi)=\frac{\alpha^{4} k_{\mathrm{v}} \rho_{\mathrm{c}}}{3 w^{2 / 3} c_{\infty}} n_{i}(x, t)
$$

With these re-scaled variables, dimensionless population balance equations are obtained that read as:

$$
\begin{aligned}
\frac{\partial f_{i}(w, \xi)}{\partial \xi}= & -\frac{\partial\left[G_{\mathrm{w}}\left(w, S_{i}\right) f_{i}(w, \xi)\right]}{\partial w} \\
& +\mathcal{A}_{1}\left[\frac{1}{2} \int_{0}^{w} A_{\mathrm{w}}(\epsilon, w-\epsilon) f_{i}(\epsilon, \xi) f_{i}(w-\epsilon, \xi) \mathrm{d} \epsilon-f_{i}(w, \xi) \int_{0}^{\infty} A_{\mathrm{w}}(\epsilon, w) f_{i}(\epsilon, \xi) \mathrm{d} \epsilon\right] \\
& +\mathcal{B}\left[\int_{w}^{\infty} B_{\mathrm{w}}(\epsilon) F_{\mathrm{w}}(\epsilon, w) f_{i}(\epsilon, \xi) \mathrm{d} \epsilon-B_{\mathrm{w}}(w) f_{i}(w, \xi)\right] \quad \text { with } i \in\{\mathrm{D}, \mathrm{L}\}
\end{aligned}
$$

where the functions appearing in the constitutive equations are now dimensionless (subscript w) and where the dimensionless parameter groups representing the agglomeration intensity $\left(\mathcal{A}_{1}\right)$ and breakage intensity $(\mathcal{B})$ have been segregated out of the constitutive equations to highlight them. All dimensionless constitutive equations are summarized in Table 2. 
Table 2: Dimensionless constitutive equations ${ }^{a}$

\begin{tabular}{ll}
\hline mechanism & equation \\
\hline solubility & $S_{\star}(w)=\exp \left(\frac{1}{w^{1 / 3}}\right)$ \\
growth and dissolution & $G_{\mathrm{w}}\left(w, S_{i}\right)=w^{2 / 3}\left(S_{i}-S_{\star}(w)\right)$ \\
breakage & $B_{\mathrm{w}}(w)=w^{k_{\mathrm{b}, 2} / 3}$ \\
& $F_{\mathrm{w}}(\epsilon, w)=\left(2 k_{\mathrm{f}}+1\right)\left(\frac{2}{\epsilon}\right)^{2 k_{\mathrm{f}}+1}\left(w-\frac{\epsilon}{2}\right)^{2 k_{\mathrm{f}}}$ \\
& $A_{\mathrm{w}}(\epsilon, w)=\frac{\left(\epsilon^{1 / 3}+w^{1 / 3}\right)^{3}}{1+\mathcal{A}_{2} w_{\mathrm{eq}}(\epsilon, w)} \quad$ with $\quad \mathcal{A}_{2}=k_{\mathrm{a}, 2} \alpha^{2}$ \\
& $w_{\mathrm{eq}}(\epsilon, w)=\frac{(\epsilon w)^{2 / 3}}{\left(\epsilon^{2}+w^{2}-\epsilon w\right)^{1 / 3}}$ \\
\hline
\end{tabular}

${ }^{a}$ In the fragment size distribution, $F_{\mathrm{w}}(\epsilon, w), \epsilon$ is the dimensionless volume of the original particle, while $w$ represents the dimensionless volume of the fragment.

For the dimensionless mass balances, one obtains:

$$
\frac{\mathrm{d} S_{i}}{\mathrm{~d} \xi}=-\int_{0}^{\infty} G_{\mathrm{w}}\left(\epsilon, S_{i}\right) f_{i}(\epsilon, \xi) \mathrm{d} \epsilon+\mathcal{R}\left(S_{j}-S_{i}\right) \quad \text { with } \quad i, j \in\{\mathrm{D}, \mathrm{L}\} \text { and } j \neq i
$$

Considering Eqs. (8) and (9) and Table 2, one can see that six parameters and parameter groups remain in the dimensionless model $\left(\mathcal{A}_{1}, \mathcal{A}_{2}, \mathcal{B}, k_{\mathrm{b}, 2}, k_{\mathrm{f}}\right.$, and $\left.\mathcal{R}\right)$; a reduction from the original eleven (as above). The dimensionless parameter groups, $\mathcal{A}_{1}, \mathcal{B}$, and $\mathcal{R}$, are defined as:

$$
\mathcal{A}_{1}=\frac{\alpha c_{\infty} k_{\mathrm{a}, 1}}{24 k_{\mathrm{v}} \rho_{\mathrm{c}} k_{\mathrm{g}}} ; \quad \mathcal{B}=\frac{\alpha k_{\mathrm{b}, 1}}{3 k_{\mathrm{g}}} ; \quad \mathcal{R}=\frac{k_{\mathrm{r}} \alpha}{3 k_{\mathrm{g}}}
$$

Each of these parameter groups therefore contains a ratio of kinetic prefactors of a pair of mechanisms occurring in Viedma ripening. Hence, they characterize the speed of agglomeration, breakage and racemization relative to the speed of crystal growth/dissolution. The relative speed of agglomeration, breakage, racemization and crystal growth/dissolution will impact the evolution of the overall process strongly. Hence, the majority of the results presented in this work focuses on varying these three parameter groups. Results for the remaining parameter groups are reported in the supporting information. 


\subsection{Numerical implementation}

Owing to their complexity Eqs. (8) and (9) cannot be solved analytically, but must be tackled numerically. Since the tasks outlined above involve running the process model for a large number of different kinetics and operating conditions (such as the initial enantiomeric excess, suspension density etc.) an efficient and robust numerical scheme to solve the equations is paramount. This can for example be accomplished by formulating Eq. (8) as a volume conservation equation. To do this, Eq. (8) is multiplied with $w$ :

$$
\begin{aligned}
w \frac{\partial f_{i}(w, \xi)}{\partial \xi}= & -w \frac{\partial}{\partial w}\left[G_{\mathrm{w}}\left(w, S_{i}\right) f_{i}(w, \xi)\right] \\
& +\mathcal{A}_{1}\left[\frac{w}{2} \int_{0}^{w} A_{\mathrm{w}}(\epsilon, w-\epsilon) f_{i}(\epsilon, \xi) f_{i}(w-\epsilon, \xi) \mathrm{d} \epsilon-w f_{i}(w, \xi) \int_{0}^{\infty} A_{\mathrm{w}}(\epsilon, w) f_{i}(\epsilon, \xi) \mathrm{d} \epsilon\right] \\
& +\mathcal{B}\left[w \int_{w}^{\infty} B_{\mathrm{w}}(\epsilon) F_{\mathrm{w}}(\epsilon, w) f_{i}(\epsilon, \xi) \mathrm{d} \epsilon-w B_{\mathrm{w}}(w) f_{i}(w, \xi)\right] \quad \text { with } \quad i \in\{\mathrm{D}, \mathrm{L}\}
\end{aligned}
$$

Using the product rule on the first term on the right hand side of Eq. (11) and reformulating the birth and death terms of agglomeration and breakage as flux terms along $w$-direction ${ }^{34-36}$,

$$
\begin{aligned}
\frac{\partial\left[w f_{i}(w, \xi)\right]}{\partial \xi}= & -\frac{\partial\left[w G_{\mathrm{w}}\left(w, S_{i}\right) f_{i}(w, \xi)\right]}{\partial w}+G_{\mathrm{w}}\left(w, S_{i}\right) f_{i}(w, \xi) \\
& -\mathcal{A}_{1} \frac{\partial}{\partial w} \int_{0}^{w} \int_{w-\epsilon}^{\infty} \epsilon A_{\mathrm{w}}(\tau, \epsilon) f_{i}(\tau, \xi) f_{i}(\epsilon, \xi) \mathrm{d} \tau \mathrm{d} \epsilon \\
& +\mathcal{B} \frac{\partial}{\partial w} \int_{w}^{\infty} \int_{0}^{w} \tau F_{\mathrm{w}}(\epsilon, \tau) B_{\mathrm{w}}(\epsilon) f_{i}(\epsilon, \xi) \mathrm{d} \tau \mathrm{d} \epsilon \quad \text { with } \quad i \in\{\mathrm{D}, \mathrm{L}\}
\end{aligned}
$$

The supporting information shows in detail that Eq. (12) is equivalent to Eq. (11) through a series of mathematical transformations. Eq. (12) is solved within a truncated computational domain, $\Lambda \in\left[0, w_{\max }\right]$ with a sufficiently large $w_{\max }$. Reflecting this and re-formulating the population balance equation in terms of the conserved quantity, $g_{i}(w, \xi)=w f_{i}(w, \xi)$ (the 
dimensionless PSD weighted with the dimensionless volume), one obtains:

$\frac{\partial g_{i}(w, \xi)}{\partial \xi}=-\frac{\partial \mathcal{F}_{\mathrm{G}, i}(w, \xi)}{\partial w}-\frac{\partial \mathcal{F}_{\mathrm{A}, i}(w, \xi)}{\partial w}+\frac{\partial \mathcal{F}_{\mathrm{B}, i}(w, \xi)}{\partial w}+\frac{G_{\mathrm{W}}\left(w, S_{i}\right) g_{i}(w, \xi)}{w} \quad$ with $\quad i \in\{\mathrm{D}, \mathrm{L}\}$

where truncated growth, agglomeration and breakage fluxes were introduced:

$$
\begin{aligned}
& \mathcal{F}_{\mathrm{G}, i}(w, \xi)=G_{\mathrm{w}}\left(w, S_{i}\right) g_{i}(w, \xi) \\
& \mathcal{F}_{\mathrm{A}, i}(w, \xi)=\mathcal{A}_{1} \int_{0}^{w} \int_{w-\epsilon}^{w_{\max }} A_{\mathrm{w}}(\tau, \epsilon) \frac{g_{i}(\tau, \xi)}{\tau} g_{i}(\epsilon, \xi) \mathrm{d} \tau \mathrm{d} \epsilon \\
& \mathcal{F}_{\mathrm{B}, i}(w, \xi)=\mathcal{B} \int_{w}^{w_{\max }} \int_{0}^{w} \tau F_{\mathrm{w}}(\epsilon, \tau) B_{\mathrm{w}}(\epsilon) \frac{g_{i}(\epsilon, \xi)}{\epsilon} \mathrm{d} \tau \mathrm{d} \epsilon
\end{aligned}
$$

In this work, the breakage and agglomeration fluxes occurring in Eq. (14) are approximated following earlier works ${ }^{34,36}$ and the growth flux is handled with a high resolution finite volume scheme first reported by Koren ${ }^{37}$. The resulting ordinary differential equations are then integrated using solvers of the Matlab ODE suite ${ }^{38}$. Full details about the discretization and of the computational domain are reported in the supporting information. For most parameter combinations investigated the outlined numerical scheme is very efficient with the majority of simulations taking in the order of a minute to complete on a standard desktop computer.

\section{Results}

As laid out in Section 2, part of the (nearly) enantiopure product made in one batch is held back and mixed with new racemic material to form the starting material of a new batch, while the remaining part of the product is harvested. The amount of product held back can therefore be used to adjust the initial solid state enantiomeric excess and therefore the productivity of the process. In order to investigate which initial enantiomeric excess leads to the highest achievable productivity and how this optimum depends on the kinetics and remaining operating parameters, sets of simulations were performed where the kinetics of all 
phenomena involved, the suspension density, as well as the size distribution of the particles to be deracemized were altered. An overview of simulation sets performed for this work is shown in Table 3. The results gathered from these simulations are analyzed regarding the following aspects: first, it will be shown that cyclic steady states are indeed attained when performing batch campaigns of deracemization processes. Their properties, as well as their implications will be highlighted in Section 4.1. The influence of the size of the racemic crystals added to each new batch will be discussed in Section 4.2. It will be shown exemplarily for one set of kinetics how process productivity depends on the initial enantiomeric excess chosen and how an optimum in process productivity can be found (Section 4.3). Finally, such optimizations will be carried out for a variety of kinetics and at different suspension densities (Section 4.4) and general trends will be identified. 
Table 3: Overview of simulations performed $\mathrm{d}^{\mathrm{a}, \mathrm{b}, \mathrm{c}}$

\begin{tabular}{|c|c|c|c|c|c|c|c|c|}
\hline sim. set & $\mathcal{A}_{1}$ & $\mathcal{A}_{2}$ & $\mathcal{B}$ & $k_{\mathrm{b}, 2}$ & $k_{\mathrm{f}}$ & $\mathcal{R}$ & $\rho_{\mathrm{s}}$ & size \\
\hline 1 & $10^{5}-10^{10}$ & $10^{5}$ & $10^{-6}-10^{-2}$ & 1 & 10 & $10^{-3}-10^{0}$ & $c_{\infty}$ & M \\
\hline 2 & $10^{5}-10^{10}$ & $10^{5}$ & $10^{-6}-10^{-2}$ & 1 & 10 & $10^{0}$ & $c_{\infty}$ & S \\
\hline 3 & $10^{5}-10^{10}$ & $10^{5}$ & $10^{-6}-10^{-2}$ & 1 & 10 & $10^{0}$ & $c_{\infty}$ & $\mathrm{L}$ \\
\hline 4 & $10^{5}-10^{10}$ & $10^{5}$ & $10^{-6}-10^{-2}$ & 1 & 10 & $10^{0}$ & $0.2 c_{\infty}$ & M \\
\hline 5 & $10^{5}-10^{10}$ & $10^{5}$ & $10^{-6}-10^{-2}$ & 1 & 10 & $10^{0}$ & $5 c_{\infty}$ & M \\
\hline 6 & $10^{5}-10^{10}$ & $10^{5}$ & $10^{-6}-10^{-2}$ & 1 & 2 & $10^{0}$ & $c_{\infty}$ & M \\
\hline 7 & $10^{5}-10^{10}$ & $10^{5}$ & $10^{-6}-10^{-3}$ & 1 & 5 & $10^{0}$ & $c_{\infty}$ & $\mathrm{M}$ \\
\hline 8 & $10^{5}-10^{10}$ & $10^{5}$ & $10^{-6}-10^{-2}$ & 1.5 & 10 & $10^{0}$ & $c_{\infty}$ & M \\
\hline 9 & $10^{5}-10^{10}$ & $10^{5}$ & $10^{-6}-10^{-2}$ & 2 & 10 & $10^{0}$ & $c_{\infty}$ & M \\
\hline
\end{tabular}

${ }^{a}$ For $\mathcal{A}_{1}, \mathcal{B}$ and $\mathcal{R}$ the simulated values are put at each whole order of magnitude within the range given, e.g., $\mathcal{R}=\left\{10^{-3}, 10^{-2}, 10^{-1}, 1\right\}$ for simulation set 1 .

b Size refers to the average size of racemic material added to each new batch; small (S): $\mu=20 \mu \mathrm{m}$, medium (M): $\mu=100 \mu \mathrm{m}$, large $(\mathrm{L}): \mu=500 \mu \mathrm{m}$. For these PSDs the standard deviation is $\sigma=0.2 \mu$ (see Eq. (15)).

c The results of simulation sets $6-9$ are shown in the supporting information, because they show a similar qualitative behavior to the conditions screened thoroughly in simulation set 1.

\subsection{Attainment of cyclic steady states}

For each combination of kinetic parameters and operating parameters (overall suspension density, initial enantiomeric excess in the solid phase) multiple batches were simulated to attain the cyclic steady state mentioned in Section 2. The first batch was always carried out with a starting enantiomeric excess in the solid phase of $2 \%$ and a saturated (racemic) liquid 
phase. Subsequent batches were carried out by keeping part of the solids of the previous batch present, so that a desired initial solid enantiomeric excess is achieved. Furthermore, all of the mother liquor from the previous batch was reused (at whatever conditions the solution was at the end of the previous batch). All simulations were run until a solid phase enantiomeric excess of $99.9 \%$ was achieved.

The newly added particles for each batch were assumed to follow a normal (Gaussian) distribution in the $x$ coordinate with a mean $\mu$ and standard deviation $\sigma(\sigma=0.2 \mu$ was specified in all cases). Reflecting that the initial particle size distribution for each batch is the sum of the newly added particles, as well as part of the product from the previous batch, the initial particle size distributions can be specified as:

$$
n_{i, m}(x, t=0)=\frac{a_{i, m}}{(2 \pi)^{1 / 2} k_{\mathrm{v}} \rho_{\mathrm{c}} \mu \sigma\left(\mu^{2}+3 \sigma^{2}\right)} \exp \left(-\frac{(x-\mu)^{2}}{2 \sigma^{2}}\right)+b_{i, m} n_{i, m-1}\left(x, t=t_{\mathrm{f}, m-1}\right)
$$

where $m$ is the (integer) batch number and $t_{\mathrm{f}, m-1}$ is the time that was required to reach the desired enantiomeric excess in the previous batch. The corresponding dimensionless particle size distributions can be written as:

$f_{i, m}(w, \xi=0)=\frac{a_{i, m}}{3(2 \pi)^{1 / 2} c_{\infty} \kappa^{1 / 3} \nu^{1 / 3}\left(\nu^{2 / 3}+3 \kappa^{2 / 3}\right) w^{2 / 3}} \exp \left(-\frac{\left(w^{1 / 3}-\nu^{1 / 3}\right)^{2}}{2 \kappa^{2 / 3}}\right)+b_{i, m} n_{i, m-1}\left(x, \xi=\xi_{\mathrm{f}, m-1}\right)$

where $\nu=\mu^{3} / \alpha^{3}, \kappa=\sigma^{3} / \alpha^{3}$ and $\xi_{\mathrm{f}, m-1}$ is the dimensionless equivalent to $t_{\mathrm{f}, m-1}$.

For the first batch to be carried out, the weighting constants $a_{i, m}$ and $b_{i, m}$ are given by:

$$
\begin{array}{ll}
a_{\mathrm{D}, 1}=\frac{\rho_{\mathrm{s}}\left(1+E_{0,1}\right)}{2} & b_{\mathrm{D}, 1}=0 \\
a_{\mathrm{L}, 1}=\frac{\rho_{\mathrm{s}}\left(1-E_{0,1}\right)}{2} & b_{\mathrm{L}, 1}=0
\end{array}
$$

where $\rho_{\mathrm{s}}$ is the overall suspension density and $E_{0, m}$ is the desired initial solid phase enantiomeric excess for batch $m$. Both quantities can be chosen as operating parameters in the 
proposed batch campaign scheme. The overall suspension density is defined as:

$$
\begin{aligned}
\rho_{\mathrm{s}} & =k_{\mathrm{v}} \rho_{\mathrm{c}}\left(\int_{0}^{\infty} x^{3} n_{\mathrm{D}}(x, t) \mathrm{d} x+\int_{0}^{\infty} x^{3} n_{\mathrm{L}}(x, t) \mathrm{d} x\right) \\
& =c_{\infty}\left(\int_{0}^{\infty} w f_{\mathrm{D}}(w, \xi) \mathrm{d} w+\int_{0}^{\infty} w f_{\mathrm{L}}(w, \xi) \mathrm{d} w\right)
\end{aligned}
$$

where definitions involving dimensional as well as dimensionless particle size distributions were given. For all subsequent batches $(m>1)$, the weighting factors are defined as:

$$
\begin{array}{rlrl}
a_{\mathrm{D}, m}=\frac{\rho_{\mathrm{s}}\left(E_{\mathrm{f}, m-1}-E_{0, \mathrm{~m}}\right)}{2 E_{\mathrm{f}, m-1}} & b_{\mathrm{D}, m} & =\frac{E_{0, m} \rho_{\mathrm{s}}\left(1+E_{\mathrm{f}, m-1}\right)}{2 E_{\mathrm{f}, m-1}} \\
a_{\mathrm{L}, m}=\frac{\rho_{\mathrm{s}}\left(E_{\mathrm{f}, m-1}-E_{0, \mathrm{~m}}\right)}{2 E_{\mathrm{f}, m-1}} & b_{\mathrm{L}, m}=\frac{E_{0, m} \rho_{\mathrm{s}}\left(1-E_{\mathrm{f}, m-1}\right)}{2 E_{\mathrm{f}, m-1}}
\end{array}
$$

Having fully specified the initial conditions, the behavior of the process in a batch campaign can be examined. While a plethora of simulations has been performed (cf. Table 3), it is illustrative to discuss one exemplary batch campaign in detail. This is done in Figure 3 with the kinetic parameters and simulation conditions given in the figure caption. In panel (a) the solid state enantiomeric excess (dashed lines) and the evolution of the dimensionless mass for both enantiomers ( $\mathrm{D}$ enantiomer as solid lines, L enantiomer as dotted lines) is shown for three consecutive batches. The dimensionless mass of enantiomer $i$ is proportional to the zeroth moment of the volume weighted dimensionless particle size distribution. The $s$-th moment of the distribution is defined as:

$$
\omega_{s, i}(\xi)=\int_{0}^{\infty} w^{s} g_{i}(w, \xi) \mathrm{d} w=\int_{0}^{\infty} w^{s+1} f_{i}(w, \xi) \mathrm{d} w
$$

The first batch was carried out with a small enantiomeric excess of $2 \%$, whereas batches 2 and 3 were carried out with $10 \%$ initial solid state enantiomeric excess. As expected, the enantiomeric excess increases with dimensionless time (note that for display purposes $-E$ is shown). This is the result of the mass of the major enantiomer (D) increasing while the 
mass of the minor enantiomer (L) is decreasing. One can see that the $E$ vs. $\xi$ curves for batch 2 and 3 are virtually identical, which indicates that a cyclic steady state was indeed reached. This impression is confirmed when the liquid phase supersaturation, as well as the full particle size distributions at the start and end of the process are considered. In panel (b) the supersaturation of both enantiomers in the liquid phase is displayed. The first batch starts at saturated conditions, but subsequently becomes slightly supersaturated for most of the batch time (at levels that would be difficult to detect experimentally). Towards the end of the batch, the supersaturation decreases again. The overall behavior is qualitatively similar to the simulation work reported by Iggland and Mazzotti ${ }^{22}$ (cf. Figure 4(c) in that work) and is typical for processes involving Ostwald ripening ${ }^{39,40}$. Note that the racemization rate chosen here is high in comparison to the crystal growth rate $(\mathcal{R}=1)$. This results in the supersaturation curves of both enantiomers to virtually overlap. In cases where the racemization rate is significantly slower $(\mathcal{R} \ll 1)$, the curves start to deviate from each other, as expected (not shown here). Panels (c) and (d) show the initial and final volume weighted dimensionless PSDs for each batch, respectively. While the initial PSDs in batch 1 are unimodal and only slightly different for both enantiomers $\left(E_{0}=2 \%\right)$, the initial PSDs for batches 2 and 3 are mixtures of the particles to be converted (with their mode at $w \approx 10^{12}$ ), as well as part of the product (smaller particles in this case) from the previous batch. Therefore, these size distributions are bimodal. In contrast, in panel $(d)$ one can see that the final PSDs for all batches are unimodal and identical. Furthermore, the PSDs of the minor enantiomer (dashed lines) have a smaller mean value and a smaller standard deviation in comparison to the PSDs of the major enantiomer (solid lines). This is not surprising, because at the end of the process almost no particles of the minor enantiomer are left and they undergo rapid dissolution. While only a few results are shown here, all simulations performed for this paper converged to the desired (D) enantiomer when the process was started with a mixture of the product and new racemic crystals.

The present work is the first time such bimodal initial distributions are studied in the 
context of Viedma ripening. However, it is useful to compare findings obtained for unimodal initial distributions ${ }^{24}$ to these results, especially since the final PSDs of the Viedma ripening process shown here are unimodal distributions. Iggland et al. ${ }^{24}$ have shown that the safest combination of parameters of the initial PSD leading to the desired enantiomer in pure form is when the average size of the desired enantiomer is larger, when its distribution is wider, and when its initial mass is higher. As one can exemplarily see from panel (d), the products obtained from the batch Viedma ripening campaign investigated here fulfill all three criteria. This was checked for all simulations carried out in this work and found to be universally true, regardless of the kinetics and the selected suspension density. These results therefore indicate that it is robust to create the initial enantiomeric excess in a batch with product crystals from the previous batch. This is obviously beneficial, because it removes the need to create seed crystals with a defined PSD for the process to deliver crystals of the desired enantiomer. Summarizing, all observations made here indicate that the proposed batch campaign is a stable cyclically operating process that is able to deliver the desired enantiomer in pure form and with a consistent PSD. 


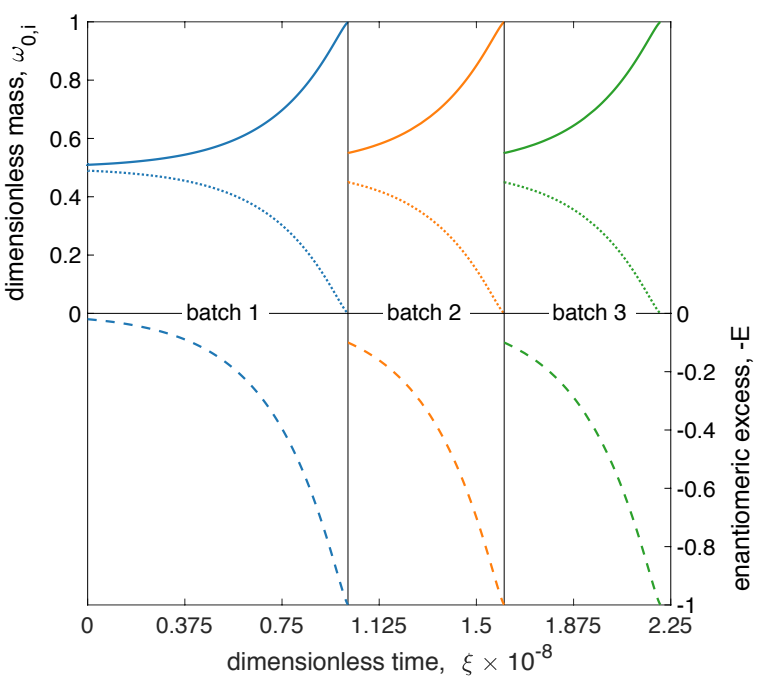

(a)

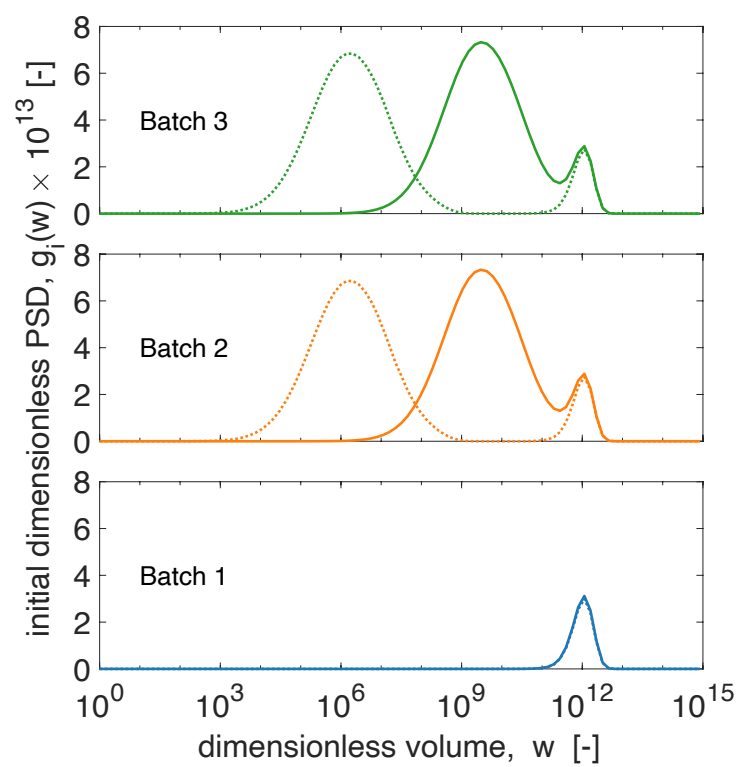

(c)

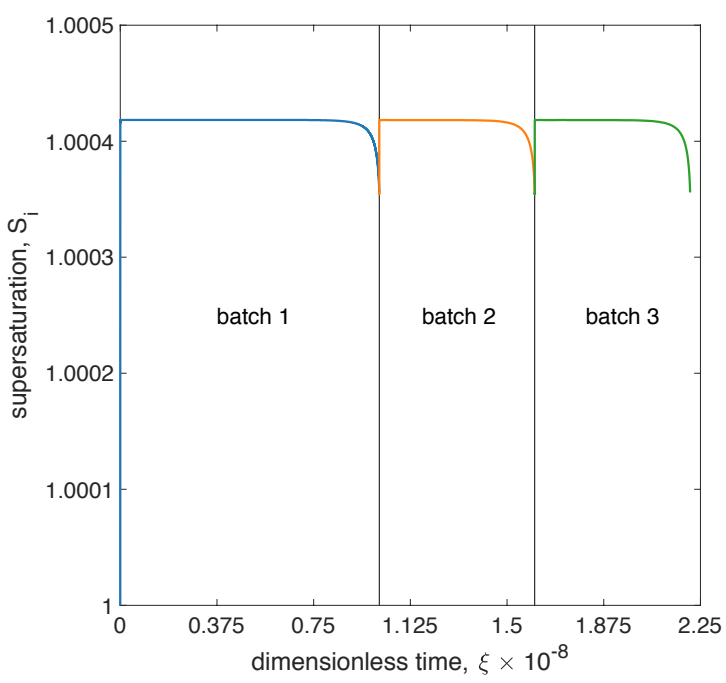

(b)

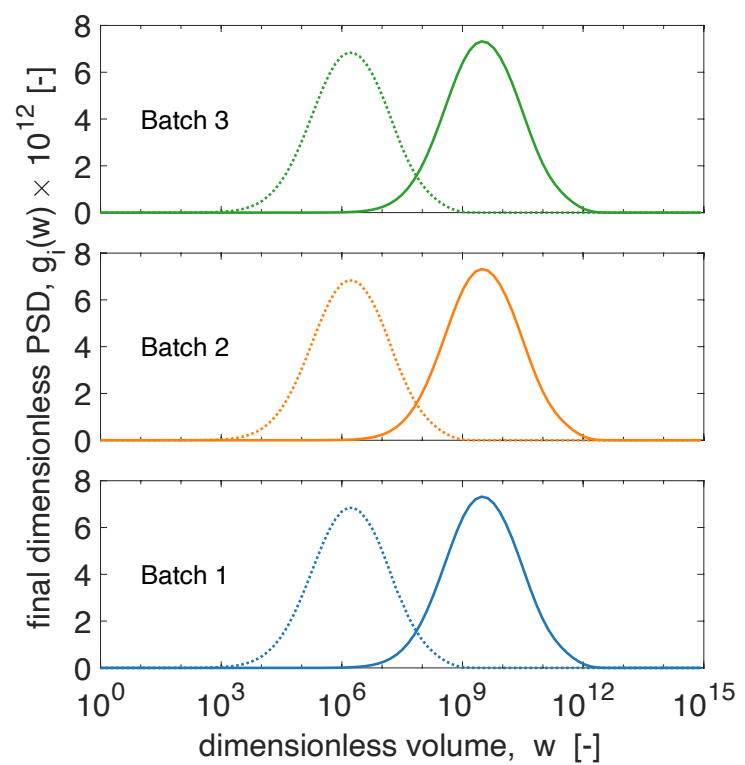

(d)

Figure 3: Output of a simulation from set 1 (cf. Table 3 ) with $\mathcal{A}_{1}=10^{9}, \mathcal{B}=10^{-5}$ and $\mathcal{R}=1$ (the values of the remaining parameters are listed in the table). Three consecutive batches are shown with different colors: blue (batch 1), orange (batch 2), green (batch 3). Curves referring to the $\mathrm{D}$ enantiomer are shown with solid lines and the $\mathrm{L}$ enantiomer is shown with dotted lines. (a) evolution of the zeroth moment of the particle size distribution (dimensionless mass) and solid phase enantiomeric excess (dashed line). (b) evolution of the supersaturation of the liquid phase. (c) Initial volume weighted particle size distributions. (d) volume weighted particle size distribution at the end of each batch. Note that in panel (b) the curves for the $\mathrm{D}$ and $\mathrm{L}$ enantiomer are overlapping. 


\subsection{Effect of the size of racemic particles to be converted}

In order to investigate the effect of the size of the racemic particles to be converted (i.e., the material that is added in each new batch) on the process behavior, series of simulations were performed with different kinetic parameters and different particle sizes (simulation sets 1-3 in Table 3). Figure 4 shows a comparison of three simulations that were performed with particles of different sizes, but with the same kinetic parameters and overall suspension density. Panel (a) of this figure shows the evolution of the dimensionless average volume of the particles of both enantiomers, $\omega_{1, i} / \omega_{0, i}$, against dimensionless time occurring for the first batch (starting with 2\% enantiomeric excess and unimodal Gaussian distributions with different mean values for the racemic material). One can see that the simulations start at substantially different average volumes, but then quickly converge towards approximately the same size (between $\xi=10^{2}$ to $\xi=10^{4}$ ). The particles of the minor enantiomer stay slightly smaller than the particles of the major enantiomer in this period. At longer times, i.e., for $\xi>10^{5}$, the sizes start diverging sharply with the particles of the minor enantiomer being driven towards dissolution and the particles of the major enantiomer becoming larger. This leads to enantiopurity at the end of the simulations. It is noteworthy that (roughly) the same time is required to reach enantiopurity regardless of the initial size of the particles. This indicates that the deracemization process is dominated by the kinetics occurring for the different mechanisms (Ostwald ripening, agglomeration, breakage), rather than the initial particle size distribution. While only a few simulations are shown here, simulation sets 1-3 include 60 different sets of kinetics (with widely varying parameters) and the same behavior was observed for all of them, i.e., whether the new material added consisted of small, medium or large particles made virtually no difference in terms of the time taken to enantiopurity. However, the different combinations of kinetic parameters resulted in different dimensionless times to achieve enantiopurity (this will be shown quantitatively in Section 4.4). Panel (b) shows the final particle size distributions of both enantiomers (minor with dashed lines; major with solid lines) for the three simulations depicted in panel (a). Clearly, not only 
the average particle volumes are virtually identical, but the whole particle size distributions, which reinforces the conclusions made above.

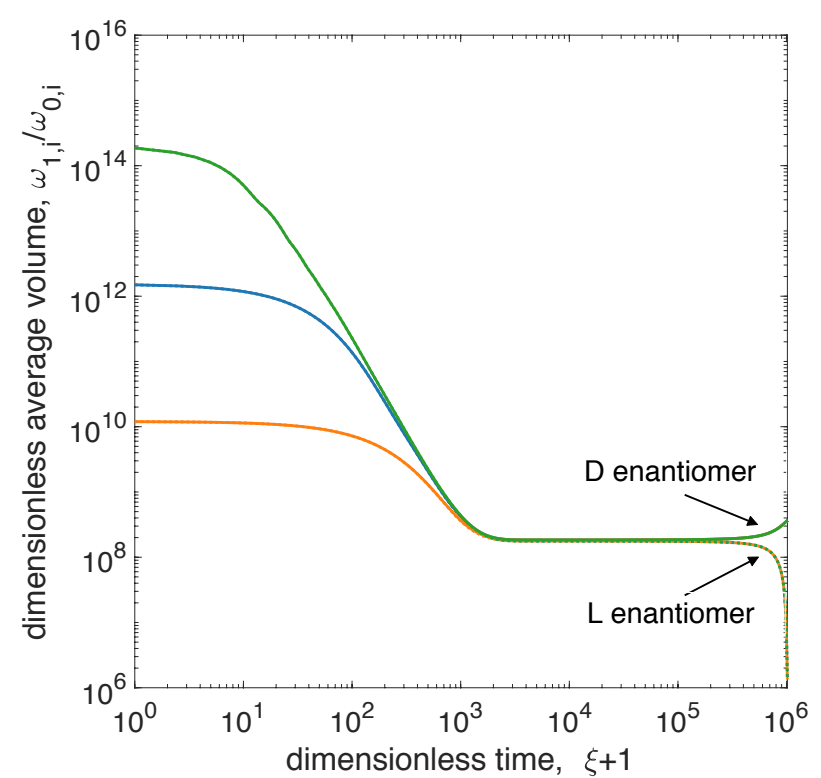

(a)

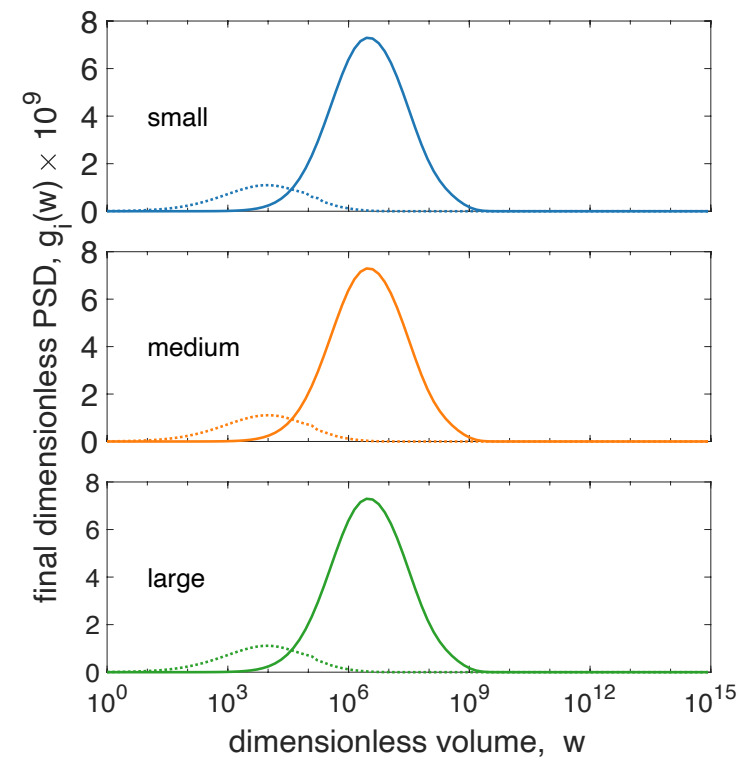

(b)

Figure 4: Average particle volume and final particle size distributions for simulations where racemic material of different sizes is added at the start of a batch. The dimensionless parameters for these simulations were $\mathcal{A}_{1}=10^{7}, \mathcal{B}=10^{-4}$, and $\mathcal{R}=1$ with the remaining parameters given in Table 3 (simulations from sets 1-3); the initial solid state enantiomeric excess was set to $2 \%$. Simulations with small, medium, and large racemic material are depicted as the blue, orange and green curves, respectively. The D enantiomer is depicted with solid lines and the L enantiomer is depicted as dotted lines. (a) Evolution of the average particle volume against dimensionless time. (b) Final particle size distributions. 


\subsection{Impact of initial enantiomeric excess on the productivity of the cyclic steady state}

Having shown that the batch campaign strategy detailed above leads to a stable cyclic steady state, it is now worthwhile to analyze the dependence of process productivity on the initial enantiomeric excess. To vary the initial enantiomeric excess, different amounts of the product of the previous batch were held back to act as starting material for the current batch. For each of the resulting initial enantiomeric excesses, the process was converged to the cyclic steady state. Panel (a) of Figure 5 shows the evolution of the dimensionless particle volume of both enantiomers (D as solid line; L as dotted line) and the evolution of the enantiomeric excess against dimensionless time for a few initial enantiomeric excesses. First, we note that the evolution of the enantiomeric excess is different from the exponential profile shown in Figure 3(a). Indeed, the shape of this curve is closely associated with the values of the kinetic constants and varies widely. For some combinations, one observes an exponential behavior (cf. Figure 3(a)), while for some values a pronounced inflection point is present (cf. Figure 5(a)). Second, one can see that - for example for the simulations starting at enantiomeric excess of 0.4 and 0.6 - the evolution of the enantiomeric excess against time is not monotonic (i.e., the enantiomeric excess first decreases, before increasing towards enantiopurity). While this is not investigated quantitatively here, the effect can be quite pronounced. This is the result of a rapid initial reshaping of the underlying particle size distributions towards smaller sizes. This in turn leads to the dissolution of a portion of them, which then increases the supersaturation in the liquid phase. If the racemization rate in the liquid phase is fast (in comparison to the other phenomena), then this process leads to more of the major/desired enantiomer being dissolved. A weak version of this behavior was already shown in the supersaturation profile in Figure 3(b), where the supersaturation at the start of each batch slightly increases, before returning to a lower value towards the end of the batch (when the particles of the major enantiomer become larger). For parameter combinations where the particles generated are smaller (high $\mathcal{B}$, low $\mathcal{A}_{1}$ ) and for fast racemization rates (high $\mathcal{R}$ ) this effect is more 
pronounced. A third observation relating to Figure 5(a) is that batches performed with a higher initial enantiomeric excess require less time to reach enantiopurity. To illustrate this, the end times of each batch are shown as vertical dotted lines in Figure 5(a). While the batch time reduces with higher initial enantiomeric excesses, the amount of product harvested per batch also reduces. Therefore, it is more meaningful to consider the productivity, which can be defined as:

$$
P=\frac{\rho_{\mathrm{s}}\left(E_{\mathrm{f}, m}-E_{0, m}\right)}{2\left(t_{\mathrm{f}, m}-t_{\mathrm{f}, m-1}\right)}
$$

where $P$ represents the mass of undesired enantiomer that has been converted to the desired enantiomer per time per process volume, i.e., the crystals of the desired enantiomer added with the racemic material are ignored in this definition. Furthermore, the definition of the productivity used here also assumes that the batch vessel(s) used do not experience downtime due to, for example, carrying out the filtration step or due to cleaning. A dimensionless productivity can also be defined:

$$
P_{\mathrm{w}}=\frac{\rho_{\mathrm{s}}\left(E_{\mathrm{f}, m}-E_{0, m}\right)}{2 c_{\infty}\left(\xi_{\mathrm{f}, m}-\xi_{\mathrm{f}, m-1}\right)}
$$

which will be used in the following when comparing process performances. The dimensionless productivity is useful here, because it is a natural way of representing the data from a dimensionless process model without assuming values for the various constants appearing in the non-dimensionalization. The initial enantiomeric excess leading to maximum productivity for a given set of kinetics and suspension density can be determined by solving an optimization problem:

$$
\underset{E_{0}}{\operatorname{maximize}} P_{\mathrm{w}}
$$

This optimization problem was solved using a golden section/parabolic interpolation method (function fminbnd in Matlab; which was done to an accuracy of 1\%). Figure 5(b) reports the process productivity for one set of kinetics and one specific value of the suspension density in dependence of the initial solid enantiomeric excess. One can see that the dimensionless 
productivity is at a maximum at $E_{0, \mathrm{opt}} \approx 0.25$ in this case. While only one such productivity vs. initial enantiomeric excess curve is shown explicitly, all of the curves generated with different kinetics and overall suspension densities showed a single maximum. The position of the maximum varies considerably for the different cases, indicating that different chemical systems will require different starting conditions to reach optimal productivity. This is investigated in the next section.

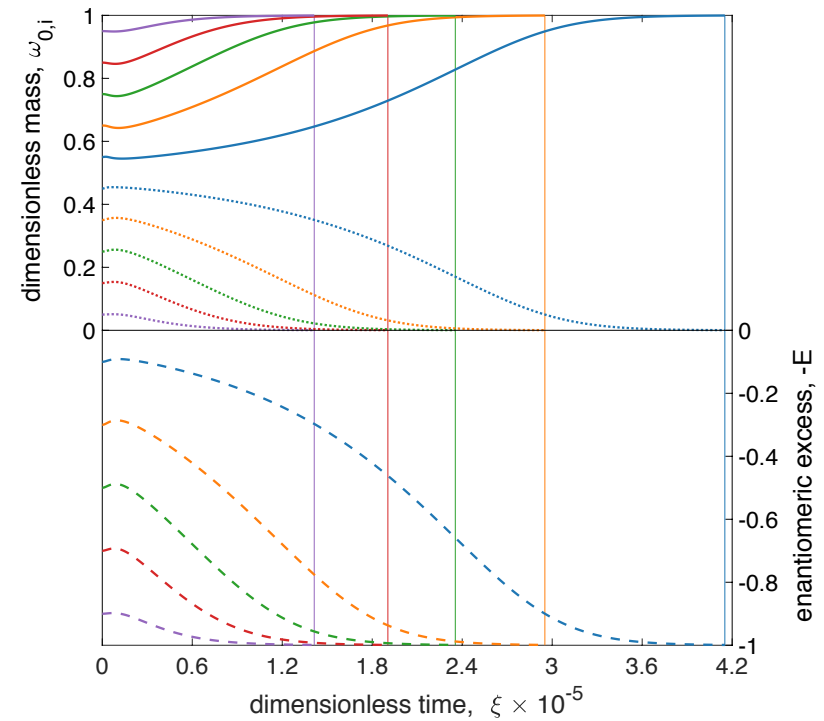

(a)

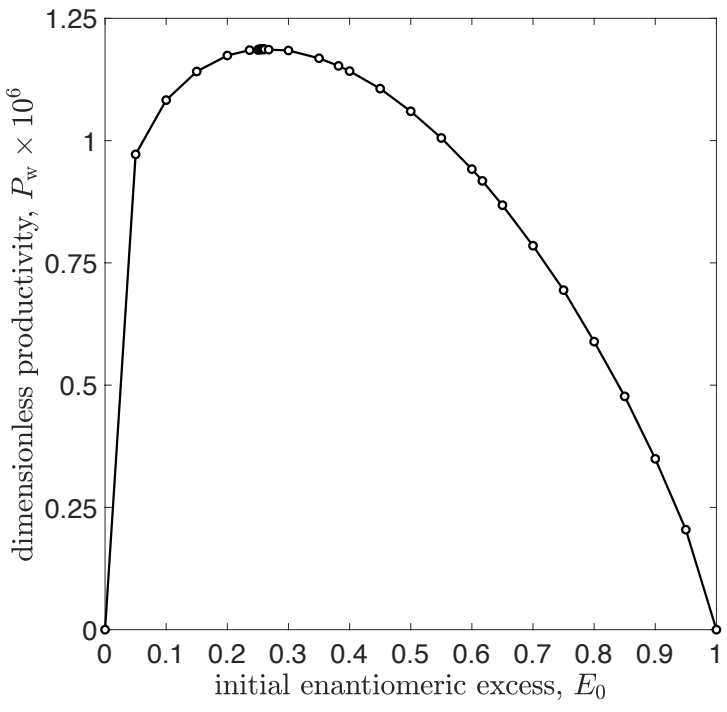

(b)

Figure 5: Simulations conducted starting from different solid phase enantiomeric excesses. The dimensionless parameters for these simulations were $\mathcal{A}_{1}=10^{5}, \mathcal{B}=10^{-5}$, and $\mathcal{R}=1$ with the remaining parameters given in Table 3 (simulation set 1). (a) Evolution of dimensionless mass of both enantiomers (solid lines: D enantiomer, dotted lines: L enantiomer) and enantiomeric excess (dashed lines) against dimensionless time. The vertical lines indicate the time required to reach a solid phase enantiomeric excess of $99.9 \%$. (b) Dimensionless productivity values (Eq. (22)) of simulations carried out at different initial solid phase enantiomeric excesses (circles). The lines in (b) are to guide the eye. The points at $0 \%$ and $100 \%$ initial enantiomeric excess stem from logical considerations, rather than simulations. 


\subsection{Effect of kinetics and suspension density on process perfor- mance}

The dependence of process productivity on the kinetics of the different mechanisms occurring in Viedma ripening and on the suspension density will be investigated here. Furthermore, it will be shown how the initial solid state enantiomeric excess leading to the optimal productivity depends on these factors. To this end, the kinetic constants appearing in the dimensionless model have been varied over several orders of magnitude, see Table 3. Figure 6(a) shows how the optimal initial enantiomeric excess obtained by solving the optimization problem in Eq. (23) varies in dependence of the agglomeration number, $\mathcal{A}_{1}$, and the breakage number, $\mathcal{B}$, when the racemization kinetics are fast in comparison to the growth kinetics $(\mathcal{R}=1)$. The optimal initial enantiomeric excess strongly depends on the extent of agglomeration occurring in the system with faster agglomeration requiring larger initial enantiomeric excesses to ensure maximum productivity. When the racemization kinetics are much slower $\left(\mathcal{R}=10^{-3}\right)$, the initial enantiomeric excess to achieve optimal process productivity increases for all combinations of kinetics, as reported in Figure 6(b). Interestingly, for the slow racemization kinetics a maximum located at high breakage numbers and intermediate agglomeration numbers appears in this figure. Furthermore, it is interesting to see that the initial enantiomeric excess required to obtain optimal productivity from the process is high in general, whereas many of the experimental studies reported in the literature utilized relatively low $(\leq 20 \%)$ initial solid enantiomeric excesses. The model study carried out here therefore predicts that there is some unharvested potential (in terms of process productivity) present in these works. This is not surprising, because many of these studies did not account for process productivity (and robustness) as a performance indicator and were therefore not optimized for it.

As one might expect, the productivity of the process (shown as a dimensionless quantity in Figure 6(c) and (d), respectively) depends strongly on the kinetics of the mechanisms involved. The results show that a higher breakage rate and lower agglomeration rate lead to 


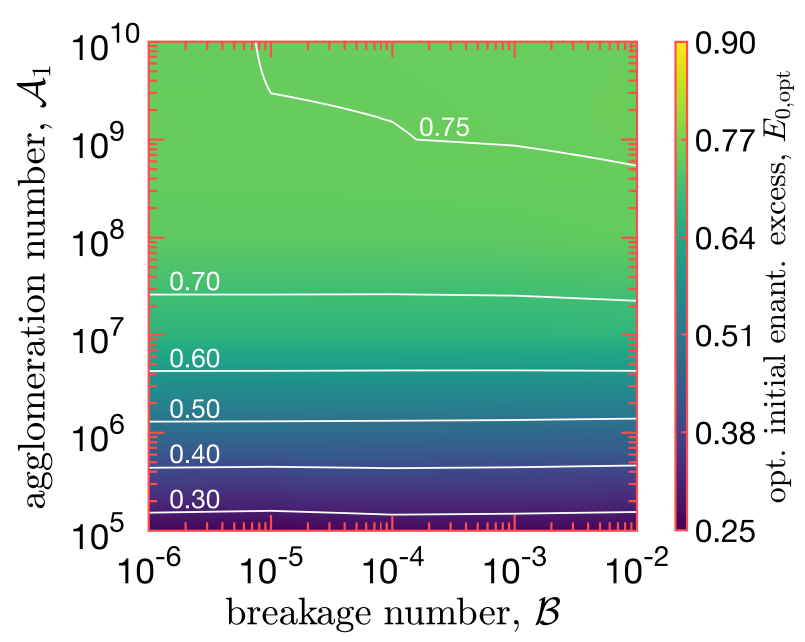

(a)

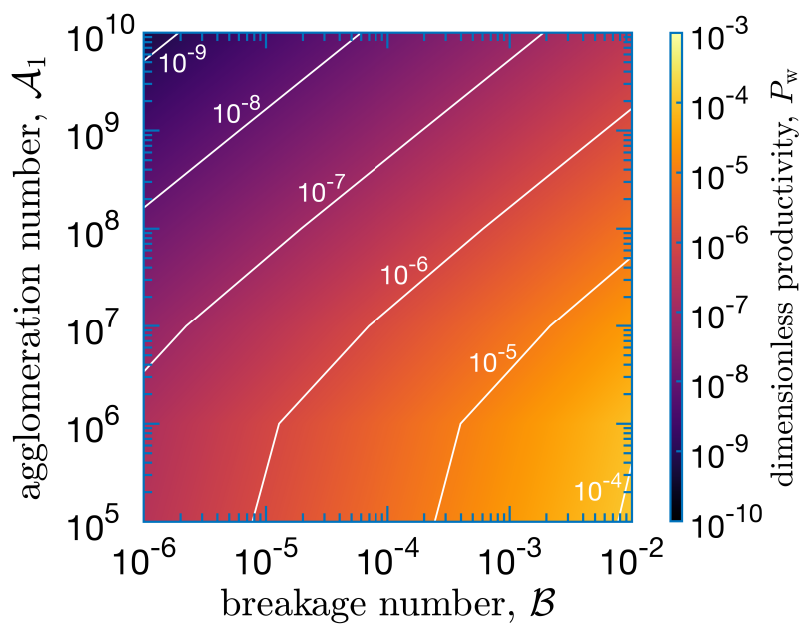

(c)

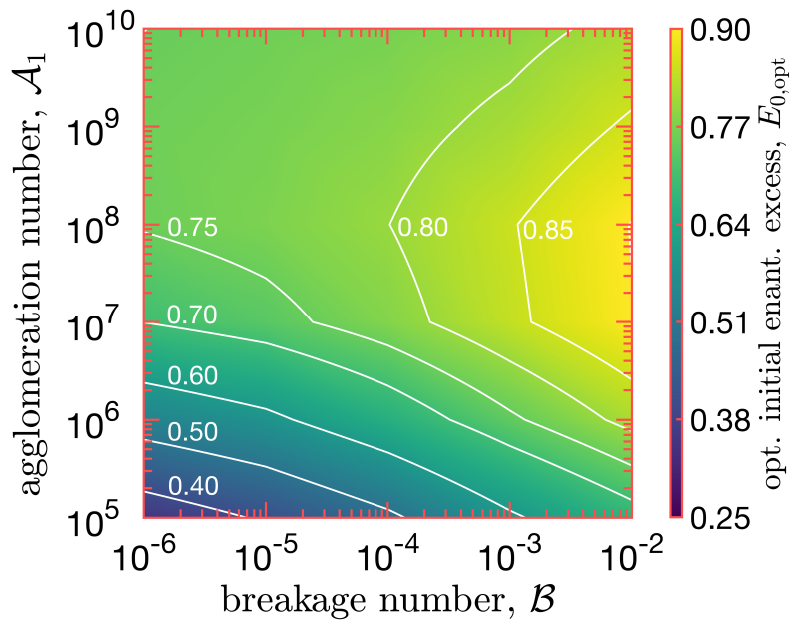

(b)

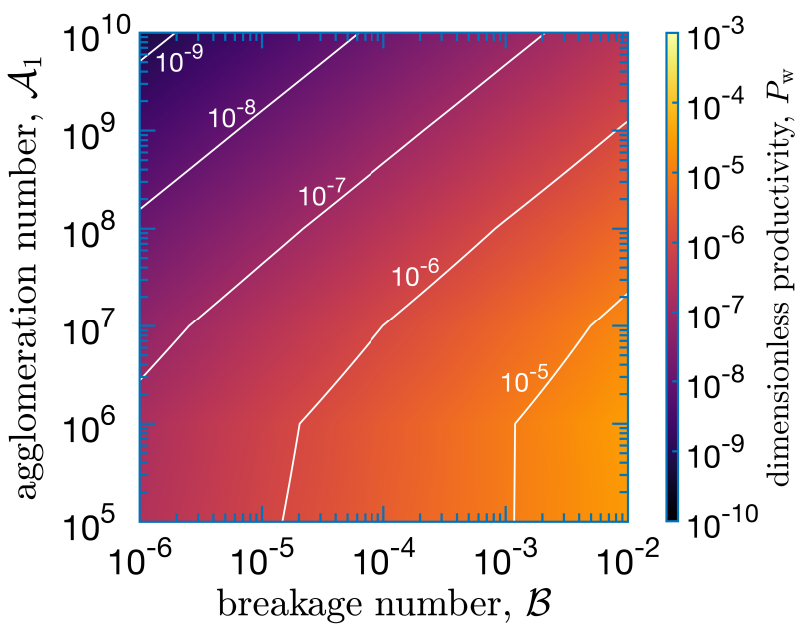

(d)

Figure 6: Optimal initial enantiomeric excess and the corresponding dimensionless productivity values for simulations carried out with different racermization numbers, agglomeration numbers $\left(\mathcal{A}_{1}\right)$ and breakage numbers $(\mathcal{B})$, corresponding to simulation set 1 in Table 3 . The left panels show results for $\mathcal{R}=1$ and the right panels show results for $\mathcal{R}=10^{-3}$. Panels (a) and (b) show the optimal initial enantiomeric excess in the solid phase. Panels (c) and (d) show the resulting dimensionless productivity values.

higher productivity when the initial enantiomeric excess has been optimized. While both agglomeration and breakage kinetics depend on material properties, the above observation is still useful from a process design perspective. For instance, the breakage rate can be tuned rather flexibly using, for example, wet milling devices (e.g., rotor stator mills). Conversely, the extent of agglomeration occurring in the system also depends on the hydrodynamics in 
the crystallizer, which provides a handle to alter them (though, to a more limited extent in comparison to breakage).

Comparing Figure 6(c) and (d) one can also see that a faster racemization reaction (higher $\mathcal{R}$ ) leads to higher dimensionless productivities if the other parameters are equivalent. This is in agreement with recent literature on polythermal deracemization processes where the racemization kinetics were quantitatively investigated ${ }^{41,42}$. The authors of both studies concluded that a higher racemization rate leads to faster deracemization if the racemization reaction is rate-limiting. However, if growth/dissolution are slow in comparison to the racemization reaction, increasing the racemization rate further would not yield any improvements in process productivity (as highlighted in Breveglieri and Mazzotti ${ }^{41}$ ). Indeed, this behavior is also found in simulations with further values of $\mathcal{R}$ reported in the supporting information, where it is evident that the productivity values start to plateau between $\mathcal{R}=10^{-2}$ and $\mathcal{R}=10^{-1}$. It is clear from the results shown so far that high breakage intensities, slow agglomeration and a high racemization rate lead to the highest productivity for the overall deracemization process. However, in practice, the solid/liquid separation step (filtration/centrifugation) occurring after the deracemization process, could contribute significantly to the overall processing time (assuming that it is not performed in parallel in a separate vessel). Since filtration times generally increase when smaller particles are involved ${ }^{43-45}$, it is useful to consider the average size of the particles generated in the process. From Figure 7 one can see that high breakage numbers and low agglomeration numbers (where the highest productivity values are located) also lead to the smallest particles. This indicates that there exists a tradeoff between a fast deracemization process and long filtration times. Hence, an overall optimized process would require that this tradeoff is quantitatively evaluated; this is, however, difficult to perform a priori and lies outside the scope of the present work.

Finally, the impact of overall suspension density on the optimal initial solid enantiomeric excess and the productivity is investigated. Figure 8 shows results of simulations carried out at five times lower and five times higher suspension density than the base case reported in 


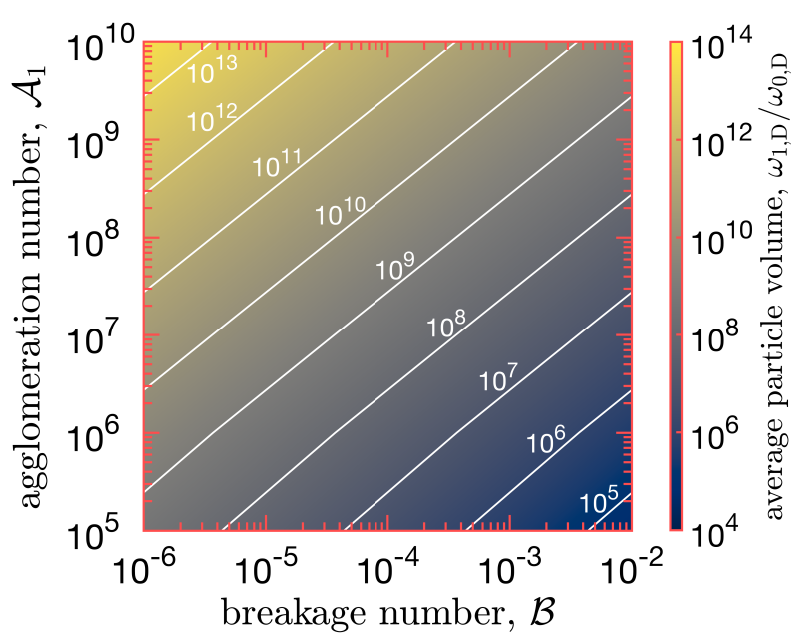

(a)

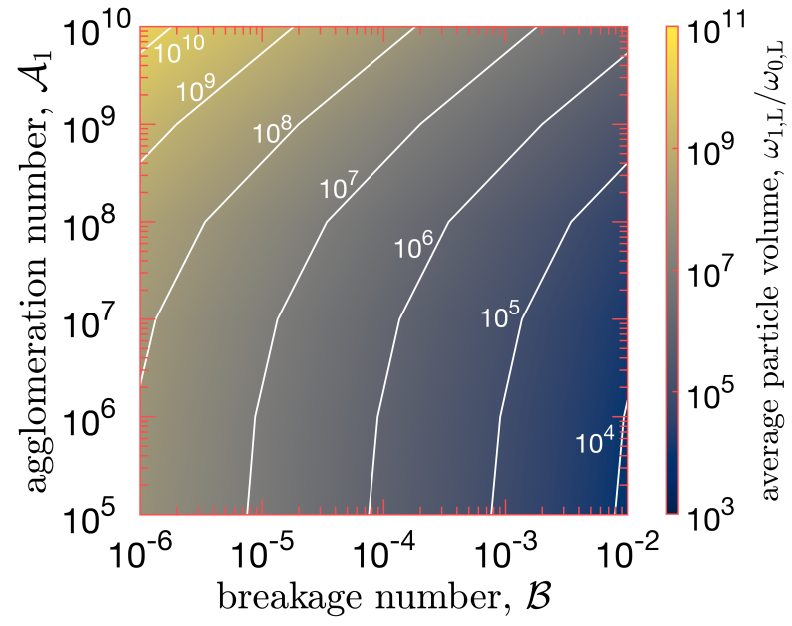

(b)

Figure 7: Average volume of the particles obtained at the end of the process in dependence of the agglomeration and breakage numbers. The racemization number was specified as $\mathcal{R}=1$; all other dimensionless parameters are given in Table 3 (simulation set 1): (a) D enantiomer (major component); (b) L enantiomer (minor component). Note that the color scales are different in the two panels and that the scale is logarithmic.

Figure 6 (simulation sets 4 and 5 in Table 3). By comparing Figure 8(a) and (b), i.e., results obtained at low and high suspension densities respectively, one can see that the initial solid phase enantiomeric excess to reach optimal productivity increases with increasing suspension density. This indicates that the deracemization process at otherwise identical kinetics becomes disproportionately slower at lower solid state enantiomeric excesses. Hence, it is beneficial to skip this part of the process by choosing a higher initial enantiomeric excess. In fact, the overall deracemization process becomes slower as well. However, the longer processing time is more than offset by the higher mass of product per batch at higher suspension density. This is evidenced in panels (c) and (d) of Figure 8 where the productivity values are clearly higher at higher suspension density (and otherwise identical kinetics).

To investigate the dependence of the optimal initial enantiomeric excess and the process productivity on the suspension density more extensively, additional results for one set of agglomeration, breakage and racemization numbers were obtained. These are reported in Figure 9. One can see that both the optimal initial solid enantiomeric excess and the productivity increase monotonically with suspension density. Therefore, the model indicates 
that high suspension densities are always beneficial in terms of productivity. That said, there are obvious practical limitations to this: at some (substance-specific) point the suspension density will become so high that the mixture inside the crystallizer becomes nigh unstirrable. Furthermore, even if the crystallizer content remains well-mixed, crystal-crystal interactions become increasingly important at high suspension densities. This, for example, can lead to non-linear breakage kinetics ${ }^{46}$. Such effects are not captured in the model expressions used here. Notwithstanding these considerations the results reported here indicate that the suspension density should be chosen high to achieve high productivities. 


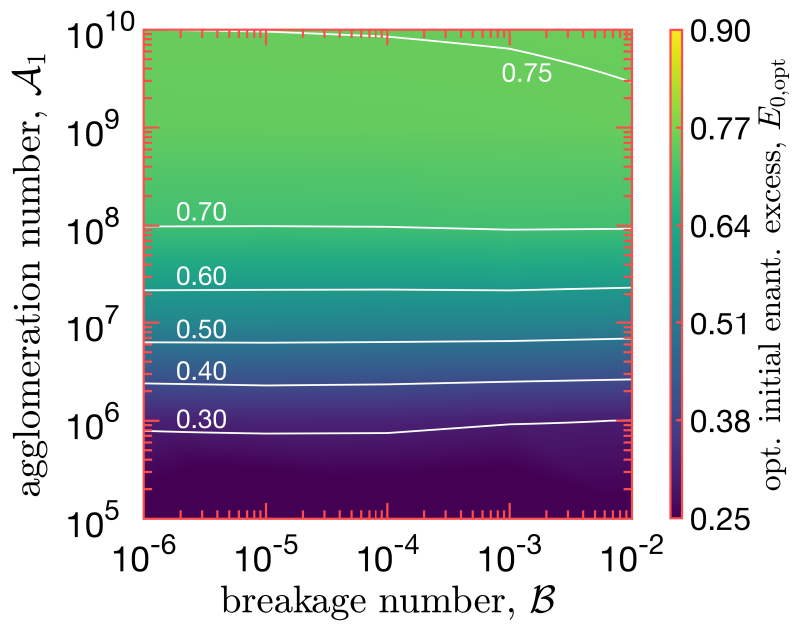

(a)

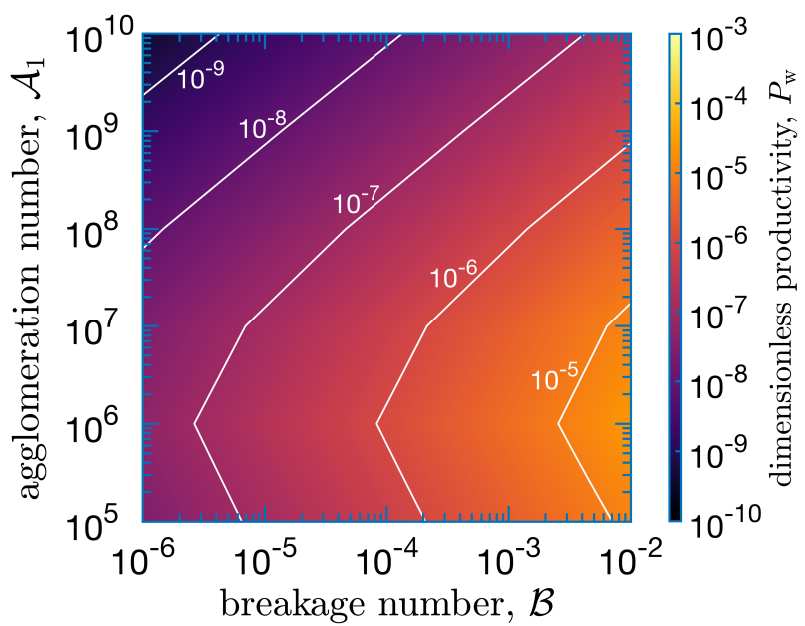

(c)

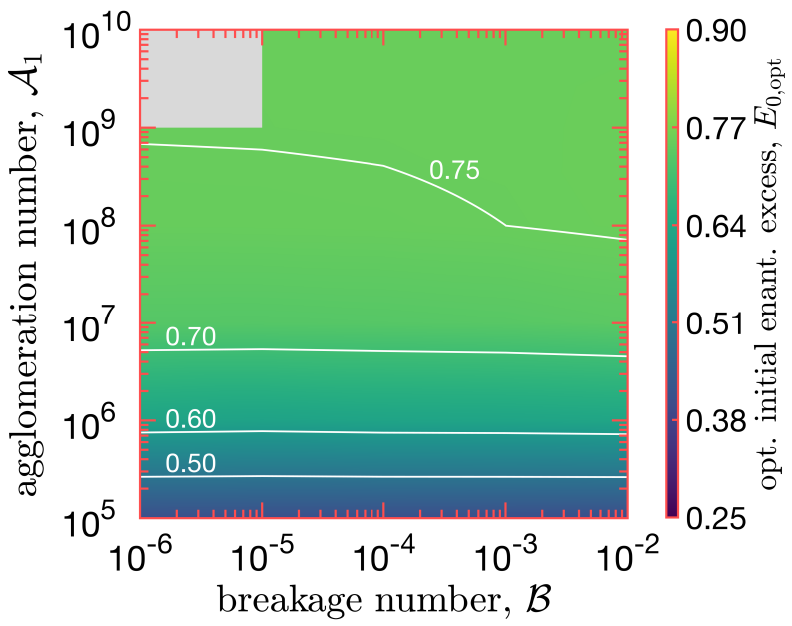

(b)

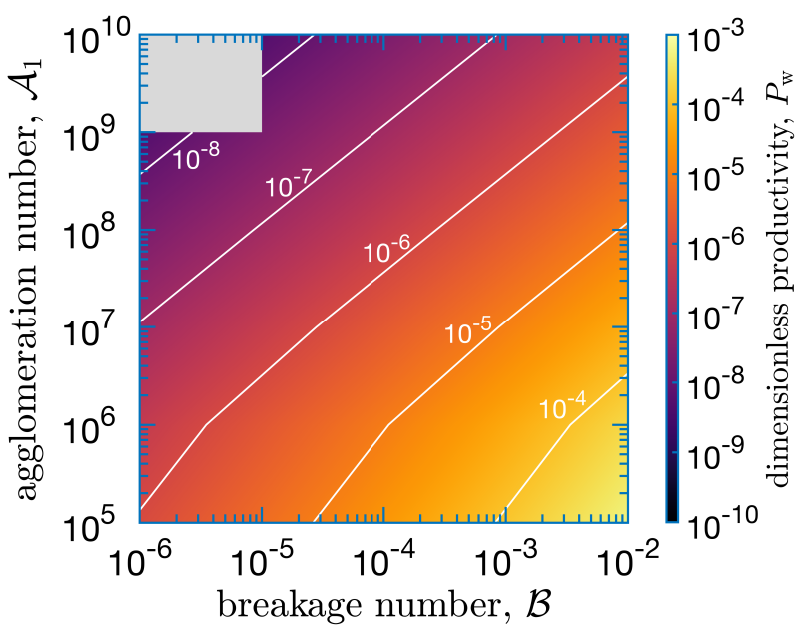

(d)

Figure 8: Optimal initial enantiomeric excess ((a) and (b)) and dimensionless productivities ((c) and (d)) for simulations carried out at low and high suspension densities (simulation sets 4 and 5 in Table 3). In the gray area in panel (b) and (d) the simulations could not be performed for numerical reasons. 


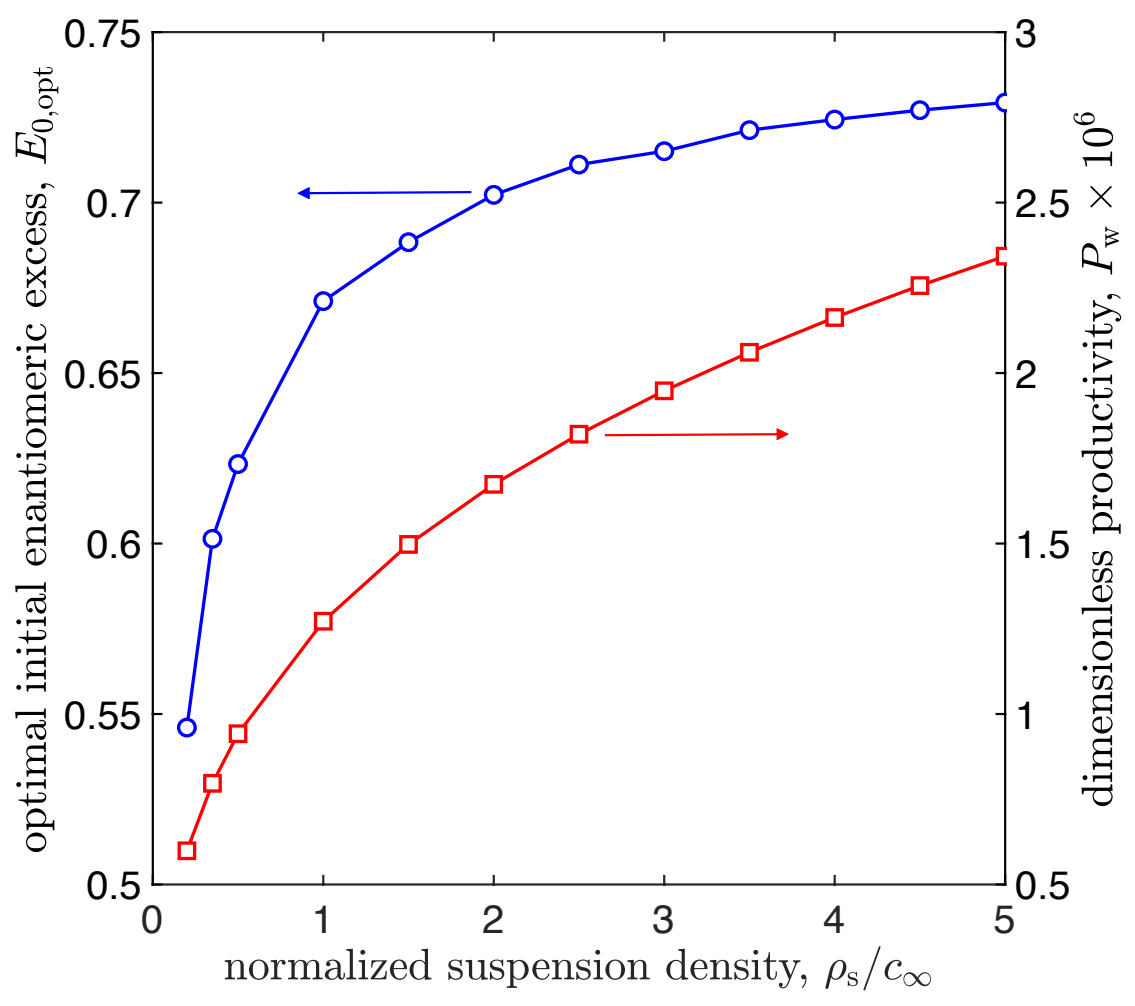

Figure 9: Simulations carried out at different suspension densities with agglomeration number $\mathcal{A}_{1}=10^{7}$, breakage number $\mathcal{B}=10^{-4}$ and racemization number $\mathcal{R}=1$. The remaining simulation parameters are as in simulation set 1 (cf. Table 3). Blue circles: optimal initial enantiomeric excess, red squares: dimensionless productivity. The lines are to guide the eye. 


\section{Conclusion}

A batch campaign strategy for isothermal deracemization processes was introduced in this work. A parametric analysis was conducted to reveal the features of the proposed batch campaign strategy using a dimensionless process model and an efficient numerical solution strategy. Thereby, it was shown that the proposed operating strategy allows for a stable cyclical operation that robustly delivers crystals of the desired enantiomer. Importantly, it was demonstrated for the first time that creating an initial enantiomeric excess with product crystals of the previous batch always drives the process towards the desired enantiomer. This is a significant result because it removes the need to generate seed crystals of a defined particle size distribution; using the product of a previous batch is obviously more convenient. The performed analysis also showed that the initial enantiomeric excess chosen for a batch has considerable impact on the process productivity. The optimal value depends strongly on the kinetics of the phenomena underpinning Viedma ripening, as well as on the overall suspension density in the crystallizer. General trends leading to productive processes were identified from the parametric analysis: in order to achieve high productivities, one should choose to operate the process at high breakage intensities, high suspension densities, as well as low agglomeration intensity.

Achieving optimal productivity for a specific substance, catalyst and solvent combination requires knowledge of the (relative) kinetics of the phenomena involved. However, determining crystallization kinetics is labour intensive in general and in particular when many mechanisms are involved (crystal growth/dissolution, agglomeration, breakage). This makes this endeavor an unappealing prospect. Alternatively, one can perform an experimental campaign where key operating parameters are varied and the above mentioned trends can be used to guide these efforts in an efficient way. Yet another alternative - a shortcut model combined with minimal data - will be introduced in the second part of this paper series. 


\section{Acknowledgments}

TV acknowledges the support of the Royal Academy of Engineering through an engineering for development research fellowship (grant no. RF1516 $15 \backslash 22$ ). This research received funding as part of the CORE project (October 2016 - September 2020) from the European Union's Horizon 2020 research and innovation programme under the Marie Sklodowska-Curie grant agreement no. 722456 CORE ITN. 


\section{Supporting Information Available}

The supporting information contains a mathematical derivation of the flux form of the dimensionless population balance equation, details about the discretization of the computational domain, as well as further simulation results (simulation sets 6-9) and a notation table.

\section{References}

(1) Lorenz, H.; Seidel-Morgenstern, A. Processes To Separate Enantiomers. Angew. Chem., Int. Ed. 2014, 53, 1218-1250.

(2) Coquerel, G. Preferential Crystallization. Top. Curr. Chem. 2006, 269, 1-51.

(3) Elsner, M.; Ziomek, G.; Seidel-Morgenstern, A. Simultaneous preferential crystallization in a coupled batch operation mode. Part II: Experimental study and model refinement. Chem. Eng. Sci. 2011, 66, 1269-1284.

(4) Levilain, G.; Eicke, M.; Seidel-Morgenstern, A. Efficient resolution of enantiomers by coupling preferential crystallization and dissolution. Part 1: Experimental proof of principle. Chem. Eng. Sci. 2012, 12, 5396-5401.

(5) Vetter, T.; Burcham, C.; Doherty, M. Separation of conglomerate forming enantiomers using a novel continuous preferential crystallization process. AIChE J. 2015, 61, 28102823.

(6) Galan, K.; Eicke, M.; Elsner, M.; Lorenz, H.; Seidel-Morgenstern, A. Continuous Preferential Crystallization of Chiral Molecules in Single and Coupled Mixed-Suspension Mixed-Product-Removal Crystallizers. Cryst. Growth Des. 2015, 15, 1808-1818.

(7) Jacques, J.; Collet, A.; Wilen, S. H. Enantiomers, racemates, and resolutions; Wiley, 1981. 
(8) Viedma, C. Chiral Symmetry Breaking During Crystallization: Complete Chiral Purity Induced by Nonlinear Autocatalysis and Recycling. Phys. Rev. Lett. 2005, 49, 84358438.

(9) Noorduin, W. L.; Izumi, T.; Millemagi, A.; Leeman, M.; Meekes, H.; Van Enckevort, W.; Kellog, R.; Kaptein, B.; Vlieg, E.; Blackmond, D. Emergence of a Single Solid Chiral State from a Nearly Racemic Amino Acid Derivative. J. Am. Chem Soc. 2008, 130, $1158-1159$.

(10) Spix, L.; Afring, A.; Meekes, H.; van Enckevort, W.; Vlieg, E. Formation of a Salt Enables Complete Deracemization of a Racemic Compound through Viedma Ripening. Cryst. Growth Des. 2014, 14, 1744-1748.

(11) Sogutoglu, L.; Steendam, R.; Meekes, H.; Vlieg, E.; Rutjes, F. Viedma ripening: a reliable crystallisation method to reach single chirality. Chem. Soc. Rev. 2015, 44, $6723-6732$.

(12) Steendam, R. E.; Verkade, J. M. M.; van Benthem, T. J. B.; Meekes, H.; van Enckevort, W. J. P.; Raap, J.; Rutjes, F. P. J. T.; Vlieg, E. Emergence of single-molecular chirality from achiral reactants. Nat. Comm. 2014, 5, 5543.

(13) Steendam, R.; Brouwer, M. C. T.; Huijs, E. M. E.; Kulka, M.; Meekes, H.; van Enckevort, W.; Raap, J.; Rutjes, F.; Vlieg, E. Enantiopure Isoindolinones through Viedma Ripening. Chem. - Eur. J. 2014, 20, 13527-1530.

(14) Noorduin, W.; van der Asdonk, P.; Bode, A.; Meekes, H.; van Enckevort, W.; Vlieg, E.; Kaptein, B.; van der Meijden, M.; Kellogg, R.; Deroover, G. Scaling Up AttritionEnhanced Deracemization by Use of an Industrial Bead Mill in a Route to Clopidogrel (Plavix). Cryst. Growth Des. 2010, 14, 908-911.

(15) Iggland, M.; Fernandez-Ronco, M.; Senn, R.; Kluge, J.; Mazzotti, M. Complete solid 
state deracemization by High Pressure Homogenization. Chem. Eng. Sci. 2014, 111, 106-111.

(16) Xiouras, C.; Van Aeken, J.; Panis, J.; ter Horst, J.; Van Gerven, T.; Stefanidis, G. Attrition-Enhanced Deracemization of NaClO3: Comparison between Ultrasonic and Abrasive Grinding. Cryst. Growth Des. 2015, 15, 5476-5484.

(17) Suwannasang, K.; Flood, A.; Coquerel, G. A Novel Design Approach To Scale Up the Temperature Cycle Enhanced Deracemization Process: Coupled Mixed Suspension Vessels. Cryst. Growth Des. 2016, 16, 6461-6467.

(18) Belletti, G.; Meekes, H.; Rutjes, F.; Vlieg, E. Role of Additives during Deracemization Using Temperature Cycling. Cryst. Growth Des. 2018, 18, 6617-6620.

(19) Breveglieri, F.; Maggioni, G.; Mazzotti, M. Deracemization of NMPA via Temperature Cycles. Cryst. Growth Des. 2018, 18, 1873-1881.

(20) Cameli, F.; Xiouras, C.; Stefanidis, G. Intensified deracemization via rapid microwaveassisted temperature cycling. CrystEngComm 2018, 20, 2897-2901.

(21) Noorduin, W. L.; van Enckevort, W.; Meekes, H.; Kaptein, B.; Kellog, R.; Tully, J.; McBride, J.; Vlieg, E. The Driving Mechanism Behind Attrition-Enhanced Deracemization. Cryst. Growth Des. 2010, 49, 8435-8438.

(22) Iggland, M.; Mazzotti, M. A Population Balance Model for Chiral Resolution via Viedma Ripening. Cryst. Growth Des. 2011, 11, 4611-4622.

(23) Xiouras, C.; Fytopoulos, A.; Ter Horst, J.; Boudouvis, A.; Van Gerven, T.; Stefanidis, G. Particle breakage kinetics and mechanisms in attrition-enhanced deracemization. Cryst. Growth Des. 2018, 18, 3051-3061.

(24) Iggland, M.; Müller, R.; Mazzotti, M. On the Effect of Initial Conditions in Viedma Ripening. Cryst. Growth Des. 2014, 14, 2488-2493. 
(25) Rajendran, A.; Paredes, G.; Mazzotti, M. Simulated moving bed chromatography for the separation of enantiomers. J. Chromatogr. A 2009, 1216, 709-738.

(26) Effendy, S.; Farooq, S.; Ruthven, D. A rigorous criterion for approach to cyclic steadystate in PSA simulations. Chem. Eng. Sci. 2017, 160, 313-320.

(27) Köllges, T.; Vetter, T. Model-based analysis of continuous crystallization/reaction processes separating conglomerate forming enantiomers. Cryst. Growth Des. 2017, 17, $233-247$.

(28) Bodàk, B.; Maggioni, G.; Mazzotti, M. Population-Based Mathematical Model of Solid-State Deracemization via Temperature Cycles. Cryst. Growth Des. 2018, 18, $7122-7131$.

(29) Randolph, A.; Larson, M. Population Balances: Theory of Particulate Processes. 2nd Edition; Academic Press, 1988.

(30) Ramkrishna, D. Population Balances - Theory and Applications to Particulate Systems in Engineering; Academic Press, 2000.

(31) Ricci, F.; Stillinger, F.; Debenedetti, P. A computational investigation of attritionenhanced chiral symmetry breaking in conglomerate crystals. J. Chem. Phys. 2013, 139, 174503.

(32) Köllges, T.; Vetter, T. Design and performance assessment of continuous crystallization processes resolving racemic conglomerates. Cryst. Growth Des. 2018, 18, 1686-1696.

(33) Li, Y.; O’Shea, S.; Yin, Q.; Vetter, T. Polymorph selection by continuous crystallization in the presence of wet milling. Cryst. Growth Des. 2019, 19, 2259-2271.

(34) Filbet, F.; Laurençot, P. Numerical simulation of the Smoluchowski coagulation equation. SIAM J. Sci. Comput. 2004, 25, 2004-2048. 
(35) Qamar, S.; Warnecke, G. Numerical solution of population balance equations for nucleation, growth and aggregation processes. Chem. Eng. Sci. 2007, 31, 1576-1589.

(36) Kumar, R.; Kumar, J. Numerical simulation and convergence analysis of a finite volume scheme for solving general breakage population balance equations. Appl. Math. Comput. 2013, 219, 5140-5151.

(37) Koren, B. In Numerical Methods Advection-Diffusion Problems; Vreugdenhil, C., Koren, B., Eds.; Vieweg: Braunschweig/Wiesbaden, 1993; Chapter 5, pp 117-138.

(38) Shampine, L.; Reichelt, M. The MATLAB ODE Suite. SIAM J. Sci. Comput. 1997, 18, $1-22$.

(39) Iggland, M.; Mazzotti, M. Population Balance Modeling with Size-Dependent Solubility: Ostwald Ripening. Cryst. Growth Des. 2012, 12, 1489-1500.

(40) Vetter, T.; Iggland, M.; Ochsenbein, D.; Hänseler, F.; Mazzotti, M. Modeling Nucleation, Growth, and Ostwald Ripening in Crystallization Processes: A Comparison between Population Balance and Kinetic Rate Equation. Cryst. Growth Des. 2013, 13, 4890-4905.

(41) Breveglieri, F.; Mazzotti, M. Role of Racemization Kinetics in the Deracemization Process via Temperature Cycles. Cryst. Growth Des. 2019, 19, 3551-3558.

(42) Oketani, R.; Hoquante, M.; Brandel, C.; Cardinael, P.; Coquerel, G. Practical Role of Racemization Rates in Deracemization Kinetics and Process Productivities. Cryst. Growth Des. 2018, 18, 6417-6420.

(43) Wakeman, R.; Tarleton, S. Solid/Liquid Separation: Principles of Industrial Filtration; Elsevier Science, 2005.

(44) am Ende, D., Ed. Chemical Engineering in the Pharmaceutical Industry: R\&D to Manufacturing; Wiley, 2010. 
(45) Perini, G.; Salvatori, F.; Ochsenbein, D.; Mazzotti, M.; Vetter, T. Filterability prediction of needle-like crystals based on particle size and shape distribution data. Sep. Purif. Technol. 2019, 211, 768-781.

(46) Capece, M.; Davé, R.; Bilgili, E. On the origin of non-linear breakage kinetics in dry milling. Powder Technol. 2015, 272, 189-203. 


\section{For Table of Contents Use Only}

Title: Designing Isothermal Batch Deracemization Processes With Optimal Productivity: I. Parametric Analysis Using A Population Balance Equation Model

Author: Thomas Vetter

TOC graphic:

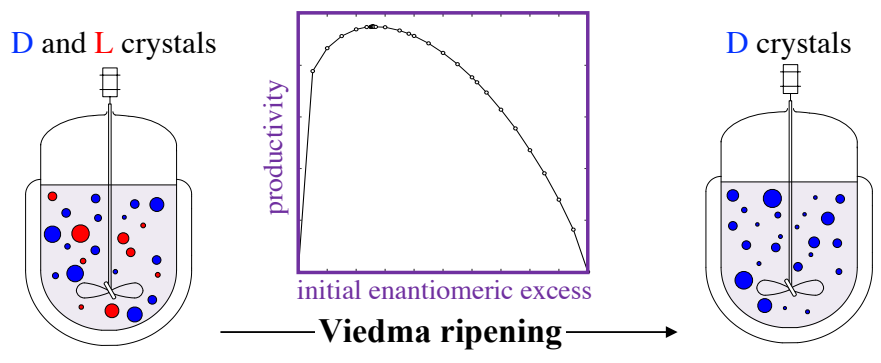

Synopsis: Isothermal batch deracemization (Viedma ripening) processes with optimal productivity are designed. A dimensionless mechanistic population balance equation model and an efficient numerical solution strategy are presented. This is used in a parametric analysis to explore the operating conditions leading to optimal process productivity in dependence of the kinetics of the mechanisms underpinning Viedma ripening. Guidelines to achieve optimal productivity are extracted from this parametric analysis. 